JOURNAL OF THE

AMERICAN MATHEMATICAL SOCIETY

Volume 21, Number 4, October 2008, Pages 1137-1168

S 0894-0347(08)00594-8

Article electronically published on January 28, 2008

\title{
ON CONFORMALLY KÄHLER, EINSTEIN MANIFOLDS
}

\author{
XIUXIONG CHEN, CLAUDE LEBRUN, AND BRIAN WEBER
}

\section{INTRODUCTION}

Recall that a Riemannian manifold $(M, h)$ is said to be Einstein if its Ricci tensor $r$ satisfies

$$
r=\lambda h
$$

for some real number $\lambda$. If, on the other hand, $M$ is equipped with an integrable almost-complex structure $J$, so that $(M, J)$ is a complex manifold, then we say that a Riemannian metric $h$ is Hermitian with respect to $J$ if $h=h(J \cdot, J \cdot)$. The purpose of this article is to prove the following:

Theorem A. If $(M, J)$ is the compact complex surface obtained from $\mathbb{C P}_{2}$ by blowing up two distinct points, then $(M, J)$ admits an Einstein metric $h$ of positive Ricci curvature which is Hermitian with respect to $J$.

The complex surface figuring in this result is diffeomorphic to $\mathbb{C P}_{2} \# 2 \overline{\mathbb{C P}}_{2}$ and can also be obtained by blowing up $\mathbb{C P}_{1} \times \mathbb{C P}_{1}$ at one point. This manifold has $c_{1}(M, J)>0$, in the sense that its first Chern class is the Kähler class of a Kähler metric; thus, it is an example of a del Pezzo surface - i.e. a Fano manifold of complex dimension 2. However, by a result of Matsushima [54, it cannot admit a KählerEinstein metric, because its automorphism group is non-reductive.

Our strategy for proving Theorem $\mathrm{A}$ was originally motivated by the work of Derdziński [12, 22] on Einstein metrics which are conformally Kähler. By extending Derdziński's results, the second author has shown elsewhere [42] that if a Hermitian metric $h$ on a compact complex surface $\left(M^{4}, J\right)$ is Einstein, then $h$ is necessarily conformal to a Kähler metric $g$, and that, unless $h$ is itself Kähler, then

- $(M, J)$ has $c_{1}>0$ and is obtained from $\mathbb{C P}_{2}$ by blowing up 1,2 , or 3 points in general position;

- $h$ has positive Ricci curvature;

- $g$ is an extremal Kähler metric in the sense of Calabi [15, 16];

- the scalar curvature $s$ of $g$ is everywhere positive; and

- after appropriate normalization, $h=s^{-2} g$.

These observations conversely motivate the proof of Theorem A, which proceeds by constructing an extremal Kähler metric $g$ with the property that $h=s^{-2} g$ is Einstein. This is done by using a weak compactness result of the first and third authors to produce large deformations of certain extremal Kähler metrics

Received by the editors May 3, 2007.

2000 Mathematics Subject Classification. Primary 53C55; Secondary 14J80, 53A30, 53C25.

The first author was supported in part by NSF grant DMS-0406346.

The second author was supported in part by NSF grant DMS-0604735.

(C)2008 American Mathematical Society Reverts to public domain 28 years from publication 
constructed by Arezzo, Pacard, and Singer [4. For a concise summary of the proof, see 92 below.

Now $\mathbb{C P}_{2} \# \overline{\mathbb{C P}}_{2}$ carries an Einstein metric originally discovered by Page [57], and it was later pointed out [12 that the Page metric is actually conformal to one of the extremal Kähler metrics constructed by Calabi [15] on the one-point blow-up $\mathbb{C P}_{2}$. As the Kähler-Einstein case has been completely solved by Aubin, Yau, and Tian [8, 74, 66, Theorem $\mathrm{A}$ exactly fills in the missing puzzle-piece needed to prove the following:

Corollary 1. Let $\left(M^{4}, J\right)$ be a compact complex surface. Then $M$ admits an Einstein metric which is Hermitian with respect to $J$ if and only if

$$
c_{1}(M, J)=\lambda[\omega]
$$

for some real constant $\lambda$ and some Kähler class $[\omega] \in H^{2}(M, \mathbb{R})$.

Theorem $\mathrm{A}$ also completes the solution of a slightly different problem. Let us instead focus on the underlying 4-manifold $M$ of a compact complex surface and, without supposing anything about the relationship between the metric and complex structure, ask when this smooth manifold admits an Einstein metric with positive $\lambda$. By the Hitchin-Thorpe inequality [12, 30, 65], the existence of such a metric implies that $M$ has $c_{1}^{2}=2 \chi+3 \tau>0$. However, the latter ensures [25, 41] that the Seiberg-Witten invariant [73] is well-defined, and the existence of a positivescalar-curvature metric then forces the invariant to vanish. But since $c_{1}^{2}>0$, the Kodaira classification [11] says that the complex surface $M$ is either rational or of general type. Since the Seiberg-Witten invariant of $M$ would be non-zero if it were of general type, we therefore conclude that $M$ can be obtained from either $\mathbb{C P}_{2}$ or a rational ruled surface by blowing up; and since $c_{1}^{2}>0$, we thus conclude that $M$ is diffeomorphic to either $\mathbb{C P}_{2} \# k \overline{\mathbb{C P}}_{2}, 0 \leq k \leq 8$, or to $S^{2} \times S^{2}$. Similarly, one can reach this same conclusion if the assumption that $M$ admits a complex structure is replaced with the hypothesis that it admits a symplectic form [51, 56]. In conjunction with the results of Tian and Yau [72, Theorem $\mathrm{A}$ therefore implies the following:

Corollary 2. Let $M$ be a smooth compact oriented 4-manifold which either admits a complex structure or admits a symplectic structure. Then $M$ carries an Einstein metric of positive scalar curvature if and only if it is orientedly diffeomorphic to either a connected sum $\mathbb{C P}_{2} \# k \overline{\mathbb{C P}}_{2}$, where $0 \leq k \leq 8$, or else to $S^{2} \times S^{2}$.

\section{StRAtegy}

We now outline the proof of Theorem $\mathrm{A}$.

Arezzo, Pacard, and Singer [4] have shown that if $\mathbb{C P}_{1} \times \mathbb{C P}_{1}$ is blown up at a point (see Figure 1), the resulting complex surface $M$ admits extremal Kähler metrics; in particular, their work shows that such metrics can be found in the Kähler classes $F_{1}+F_{2}-\epsilon E$ for any sufficiently small $\epsilon>0$, where $E$ is the Poincaré dual of the exceptional divisor introduced by blowing up and where $F_{1}$ and $F_{2}$ are the Poincaré duals of the factor $\mathbb{C P}_{1}$ 's of $\mathbb{C P}_{1} \times \mathbb{C P}_{1}$. We note in passing that the homology classes $F_{1}-E$ and $F_{2}-E$ are also represented by (-1)-curves and that blowing these two exceptional divisors down (see Figure 2) results in $\mathbb{C P}_{2}$; thus $M$ may also be described as $\mathbb{C P}_{2} \# 2 \overline{\mathbb{C P}}_{2}$. We also note that the Kähler classes we are choosing to study are ones for which $F_{1}$ and $F_{2}$ have equal areas, even though the 

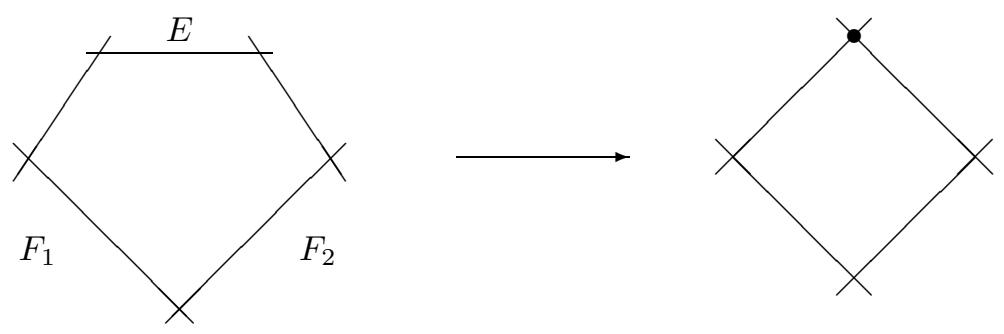

Figure 1
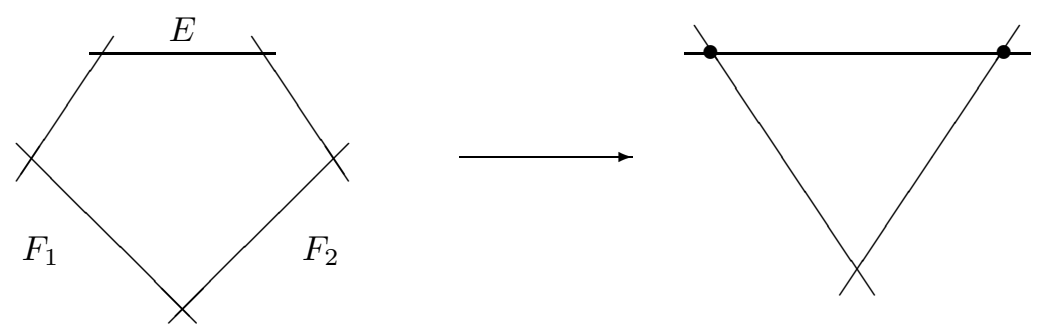

FiguRE 2

Arezzo-Pacard-Singer result would also construct extremal Kähler metrics for which the ratios of these areas is quite arbitrary. By the uniqueness of extremal Kähler metrics in a given Kähler class [19], the metrics we are considering therefore not only have an isometric $U(1) \times U(1)$-action, but also admit an additional isometric $\mathbb{Z}_{2^{-}}$ action which interchanges $F_{1}$ and $F_{2}$. This leads to major technical simplifications which will play a crucial rôle in our proof. We thus introduce the term bilaterally symmetric to describe both those Kähler classes which are invariant under the interchange $F_{1} \leftrightarrow F_{2}$, as well as the extremal Kähler metrics we will find in many such classes.

By a general result [4] proved via the inverse-function theorem, the extremal cone, consisting of the Kähler classes of all extremal Kähler metrics on $(M, J)$, is automatically open in $H^{1,1}(M, \mathbb{R})$; consequently, the set of $\epsilon$ for which the relevant Kähler class contains an extremal Kähler metric is open. As we increase $\epsilon$, we can then use the Futaki invariant to show that the value of the Calabi functional

$$
\mathcal{C}(g)=\int_{M} s_{g}^{2} d \mu_{g}
$$

on these extremal metrics initially decreases but would eventually reach a minimum and then increase if we could simply take $\epsilon$ to be sufficiently large. If we can simply arrange for $\epsilon$ to achieve a value which extremizes $\mathcal{C}(g)$, we show in 44 that the corresponding extremal metric will then actually be conformally Einstein.

Thus, the problem essentially boils down to showing that, within a certain range, the set of $\epsilon$ achieved by extremal Kähler metrics is actually closed as well as open. Our method of showing this is based on an orbifold compactness result proved elsewhere by the first and third authors [20. In order to apply this, we must first prove a uniform estimate for the Sobolev constant of the metrics involved; this is done in $\$ 5$ Next, we must show that orbifold singularities cannot form in the limit. This is done by showing that curvature can never concentrate in too small a region, since, upon rescaling, this would result in an asymptotically locally flat 
manifold which, given the topological and symmetry conditions imposed by our situation, would ultimately require the concentration of more curvature than is actually available.

\section{The Calabi functional}

If $(M, g, J)$ is an extremal Kähler metric on a compact complex surface, the Calabi functional takes the value

$$
\mathcal{C}(g)=s_{0}^{2} \int d \mu+\int\left(s-s_{0}\right)^{2} d \mu=32 \pi^{2} \frac{\left(c_{1} \cdot[\omega]\right)^{2}}{[\omega]^{2}}-\mathcal{F}(\xi,[\omega])
$$

where $s_{0}$ is the average value of the scalar curvature, $\mathcal{F}$ denotes the Futaki invariant, and $\xi=\operatorname{grad}^{1,0} s$ is the extremal vector field of the class [ $\left.\omega\right]$. It is crucial for our purposes that $\xi$ may be determined 27] up to conjugation even without knowing that an extremal metric exists. Thus, one may define a functional

$$
\mathcal{A}([\omega])=\frac{\left(c_{1} \cdot[\omega]\right)^{2}}{[\omega]^{2}}-\frac{1}{32 \pi^{2}} \mathcal{F}(\xi,[\omega])
$$

on the entire Kähler cone, independent of the existence of extremal Kähler metrics. This functional has the important property [18] that any Kähler metric $g$ in the Kähler class $[\omega]$ satisfies the curvature inequality

$$
\frac{1}{32 \pi^{2}} \int s^{2} d \mu \geq \mathcal{A}([\omega])
$$

with equality iff $g$ is an extremal metric. Notice that our normalization has been chosen so that we automatically have

$$
\mathcal{A}([\omega]) \geq c_{1}^{2}(M)
$$

for any Kähler class. This section will now begin with a discussion of the problem, first explored in 41, of finding a critical point of $\mathcal{A}$, considered as a function on the Kähler cone. To do this, we will use computations of the Futaki invariant first given in 45. for the blow-up of $\mathbb{C} P_{2}$ at $\leq 3$ points in general position. Any extremal Kähler metric is invariant [16] under a maximal compact subgroup of the identity component of the complex automorphism group, and since such subgroups are unique up to conjugation, we may simply choose one; in the present case, this means that we may consider only metrics which are invariant under the 2-torus $T^{2}$ of automorphisms of $M$ induced by

$$
\left(\left[u_{1}: u_{2}\right],\left[v_{1}: v_{2}\right]\right) \mapsto\left(\left[u_{1}: e^{i \theta} u_{2}\right],\left[v_{1}: e^{i \phi} v_{2}\right]\right),
$$

where $M$ is thought of as the blow-up of $\mathbb{C P}_{1} \times \mathbb{C P}_{1}$ at $([0: 1],[0: 1])$. We also choose only to consider bilaterally symmetric Kähler classes $[\omega]=(\beta+\varepsilon)\left(F_{1}+F_{2}\right)-\varepsilon E$ on $M=\mathbb{C P}_{2} \# 2 \overline{\mathbb{C P}}_{2}$ :

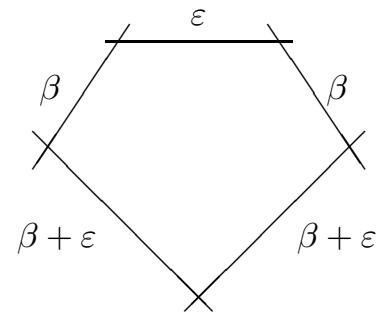


Here the term bilaterally symmetric is again used to indicate that the class in question is invariant under $F_{1} \leftrightarrow F_{2}$. The numbers $\beta$ and $\varepsilon$ respectively represent the areas of the $(-1)$-curves $F_{1}-E$ and $E$; both are thus required to be positive, but they may otherwise be taken to be completely arbitrary. Since $\mathcal{A}$ is invariant under the $\mathbb{Z}_{2}$-action $F_{1} \leftrightarrow F_{2}$ induced by interchanging the factors of $\mathbb{C P}_{1} \times \mathbb{C P}_{1}$ and is also invariant under rescaling $[\omega] \rightsquigarrow a[\omega]$, any critical point of the function

$$
f(x)=\mathcal{A}\left([1+x]\left(F_{1}+F_{2}\right)-x E\right)
$$

will yield a critical point $(\beta, \varepsilon)=(1, x)$, and conversely, up to rescaling, a critical point of $\mathcal{A}$ arises this way if and only if the relevant Kähler class is bilaterally symmetric.

Now, for any $T^{2}$-invariant, bilaterally symmetric Kähler metric, the real part of the extremal Kähler vector field $\xi$ belongs to the Lie algebra of our maximal compact subgroup $T^{2} \subset \operatorname{Aut}_{0}(M)$ and must be invariant under $F_{1} \leftrightarrow F_{2}$. Thus $\xi$ must be a multiple of the generator $\Xi$ of the $\mathbb{C}^{\times}$-action induced by the action

$$
\left(\left[u_{1}: u_{2}\right],\left[v_{1}: v_{2}\right]\right) \mapsto\left(\left[u_{1}: \zeta u_{2}\right],\left[v_{1}: \zeta v_{2}\right]\right)
$$

on $\mathbb{C P}_{1} \times \mathbb{C P}_{1}$. But $\Xi=\operatorname{grad}^{1,0} t$ for a real-valued Hamiltonian function $t$ which by symplectic reduction [4] can be shown to satisfy

$$
\begin{aligned}
{[12 \pi \omega]^{2} \int\left(t-t_{0}\right)^{2} d \mu=} & 12 \beta^{6}+72 \beta^{5} \varepsilon+138 \beta^{4} \varepsilon^{2}+120 \beta^{3} \varepsilon^{3} \\
& +54 \beta^{2} \varepsilon^{4}+12 \beta \varepsilon^{5}+\varepsilon^{6}
\end{aligned}
$$

where $t_{0}$ is the average value of $t$. On the other hand, it was shown in 45] that

$$
[\omega]^{2} \mathcal{F}(\Xi,[\omega])=4 \beta \varepsilon\left[\frac{\varepsilon^{2}}{3}+\beta \varepsilon+\beta^{2}\right] .
$$

Since $\mathcal{F}(\Xi,[\omega])=-\int\left(t-t_{0}\right)\left(s-s_{0}\right) d \mu$, an explicit formula for $\mathcal{A}$ can now be deduced by setting $\left(s-s_{0}\right)=\lambda\left(t-t_{0}\right)$ and solving for $\lambda$ to obtain

$$
\lambda=-\frac{(12 \pi)^{2} 4 \beta \varepsilon\left[\varepsilon^{2} / 3+\beta \varepsilon+\beta^{2}\right]}{12 \beta^{6}+72 \beta^{5} \varepsilon+138 \beta^{4} \varepsilon^{2}+120 \beta^{3} \varepsilon^{3}+54 \beta^{2} \varepsilon^{4}+12 \beta \varepsilon^{5}+\varepsilon^{6}}
$$

so that

$$
\begin{aligned}
-\frac{1}{32 \pi^{2}} \mathcal{F}(\xi,[\omega]) & =-\frac{1}{32 \pi^{2}} \lambda \mathcal{F}(\Xi,[\omega]) \\
& =\frac{9}{2[\omega]^{2}} \frac{\left(4 \beta \varepsilon\left[\varepsilon^{2} / 3+\beta \varepsilon+\beta^{2}\right]\right)^{2}}{12 \beta^{6}+72 \beta^{5} \varepsilon+138 \beta^{4} \varepsilon^{2}+120 \beta^{3} \varepsilon^{3}+54 \beta^{2} \varepsilon^{4}+12 \beta \varepsilon^{5}+\varepsilon^{6}}
\end{aligned}
$$

and

$$
\begin{aligned}
\mathcal{A}([\omega]) & =\frac{\left(c_{1} \cdot[\omega]\right)^{2}}{[\omega]^{2}}-\frac{1}{32 \pi^{2}} \mathcal{F}(\xi,[\omega]) \\
& =\frac{(4 \beta+3 \varepsilon)^{2}}{[\omega]^{2}}-\frac{1}{32 \pi^{2}} \mathcal{F}(\xi,[\omega])
\end{aligned}
$$

Hence

$$
\mathcal{A}([\omega])=f(x)=3\left(\frac{32+176 x+318 x^{2}+280 x^{3}+132 x^{4}+32 x^{5}+3 x^{6}}{12+72 x+138 x^{2}+120 x^{3}+54 x^{4}+12 x^{5}+x^{6}}\right)
$$




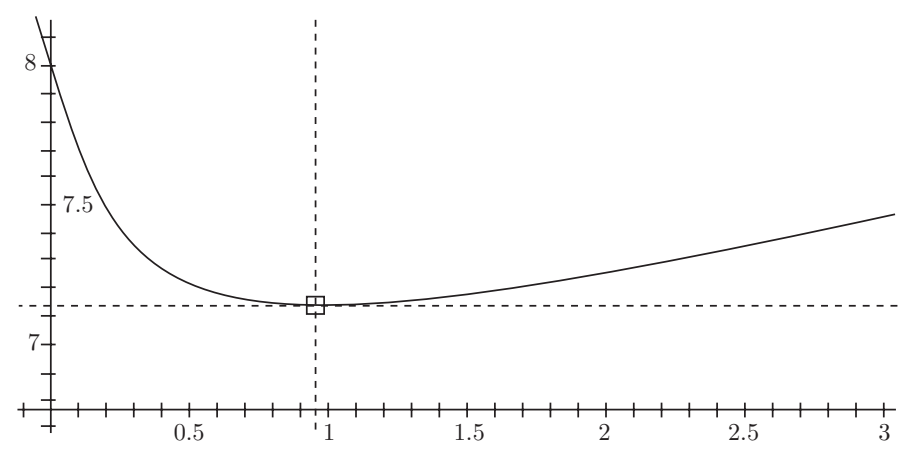

FiguRE 3

where $x=\varepsilon / \beta$. For $x>0$, technology 11 indicates that this has a unique critical point, an absolute minimum, at $x \approx 0.958$ (see Figure 3 ). However, we will need a great deal less for the purposes of our proof:

Lemma 3. There is a number $x_{0}>0$ such that the function

$$
f(x)=3\left(\frac{32+176 x+318 x^{2}+280 x^{3}+132 x^{4}+32 x^{5}+3 x^{6}}{12+72 x+138 x^{2}+120 x^{3}+54 x^{4}+12 x^{5}+x^{6}}\right)
$$

has a critical point at $x=x_{0}$ and such that $f(x)<8$ on $\left(0, x_{0}\right]$.

Proof. Notice that $f(0)=8$ and that $f^{\prime}(0)=-4<0$, so $f(x)<8$ for small positive $x$. However, $\lim _{x \rightarrow \infty} f(x)=9$, so $f^{\prime}(x)$ must be positive somewhere. We can therefore simply define $x_{0}$ to be the first positive number at which $f^{\prime}(x)=0$, since it then follows that $f$ is decreasing on $\left[0, x_{0}\right]$.

We leave it as an exercise for the interested reader to check that $f^{\prime}(1)>0$, so that in fact $x_{0}<1$. However, it turns out that we will never actually need this sort of information for the purposes of our proofs.

We would also like to know if there are values of $x=\varepsilon / \beta$ for which the scalar curvature of the corresponding Kähler metric is everywhere positive. To determine this, notice that, since the Hamiltonian $t$ generates rotations of period $2 \pi$ of $F_{1}$ and $F_{2}-E$, while leaving $E$ fixed, symplectic geometry tells us that

$$
(\beta+\varepsilon)+\beta=2 \pi\left(t_{\max }-t_{\min }\right),
$$

and hence that

$$
s_{\max }-s_{\min }=\frac{|\lambda|}{2 \pi}(2 \beta+\varepsilon) .
$$

\footnotetext{
${ }^{1}$ Figure 3 drawn using Pacific Tech's Graphing Calculator program for Mac OS X.
} 
Thus

$$
\begin{aligned}
& s_{\min }=s_{\max }-\frac{|\lambda|}{2 \pi}(2 \beta+\varepsilon) \\
& >s_{0}-\frac{|\lambda|}{2 \pi}(2 \beta+\varepsilon) \\
& =4 \pi \frac{c_{1} \cdot[\omega]}{[\omega]^{2} / 2}-\frac{|\lambda|}{2 \pi}(2 \beta+\varepsilon) \\
& =\frac{8 \pi(4 \beta+3 \varepsilon)}{2 \beta^{2}+4 \beta \varepsilon+\varepsilon^{2}}-\frac{72 \pi(2 \beta+\varepsilon) 4 \beta \varepsilon\left[\varepsilon^{2} / 3+\beta \varepsilon+\beta^{2}\right]}{12 \beta^{6}+72 \beta^{5} \varepsilon+138 \beta^{4} \varepsilon^{2}+120 \beta^{3} \varepsilon^{3}+54 \beta^{2} \varepsilon^{4}+12 \beta \varepsilon^{5}+\varepsilon^{6}} \\
& =8 \pi \beta^{-1}\left[\frac{4+3 x}{2+4 x+x^{2}}-\frac{9(2+x) 4 x\left[x^{2} / 3+x+1\right]}{12+72 x+138 x^{2}+120 x^{3}+54 x^{4}+12 x^{5}+x^{6}}\right] \\
& =8 \pi \beta^{-1} \frac{48+180 x+264 x^{2}+270 x^{3}+204 x^{4}+102 x^{5}+28 x^{6}+3 x^{7}}{\left(2+4 x+x^{2}\right)\left(12+72 x+138 x^{2}+120 x^{3}+54 x^{4}+12 x^{5}+x^{6}\right)} \\
& >0 .
\end{aligned}
$$

This proves the following:

Lemma 4. Any bilaterally symmetric extremal Kähler metric on $M=\mathbb{C P}_{2} \# 2 \overline{\mathbb{C P}}_{2}$ has strictly positive scalar curvature.

This computation also implies a $C^{0}$ estimate for the scalar curvature of such metrics. Indeed, we now have

$$
s_{\max }=s_{\min }+\frac{|\lambda|}{2 \pi}(2 \beta+\varepsilon)<s_{0}+\frac{|\lambda|}{2 \pi}(2 \beta+\varepsilon)<2 s_{0} .
$$

Letting $V=[\omega]^{2} / 2$ denote the total volume, we thus have

$$
s_{\max } V^{1 / 2}<2 s_{0} V^{1 / 2}=\frac{8 \pi c_{1} \cdot[\omega]}{\sqrt{[\omega]^{2} / 2}}=\frac{8 \pi(4 \beta+3 \varepsilon) \sqrt{2}}{\sqrt{2 \beta^{2}+4 \beta \varepsilon+\varepsilon^{2}}}<24 \pi \sqrt{2},
$$

so that we have the following:

Lemma 5. The scalar curvature of any bilaterally symmetric extremal Kähler metric $g$ on $M=\mathbb{C P}_{2} \# 2 \overline{\mathbb{C P}}_{2}$ satisfies the $C^{0}$ estimate

$$
|s| V^{1 / 2}<24 \pi \sqrt{2} .
$$

\section{The BACH TENSOR}

If $M$ is any smooth compact oriented 4-manifold, consider the conformally invariant Riemannian functional

$$
\mathcal{W}(g)=\int_{M}|W|_{g}^{2} d \mu_{g}
$$

obtained by squaring the $L^{2}$-norm of the Weyl curvature. For any smooth 1parameter family of metrics

$$
g_{t}:=g+t \dot{g}+O\left(t^{2}\right)
$$

the first variation of this functional is then given by

$$
\left.\frac{d}{d t} \mathcal{W}\left(g_{t}\right)\right|_{t=0}=-\int \dot{g}^{a b} B_{a b} d \mu
$$


where [10, 12 the Bach tensor $B$ is given by

$$
B_{a b}:=\left(\nabla^{c} \nabla^{d}+\frac{1}{2} r^{c d}\right) W_{a c b d} .
$$

The Bach tensor is automatically symmetric and trace-free, and the latter is precisely the infinitesimal version of the fact that the functional $\int|W|^{2} d \mu$ is conformally invariant. Similarly, since $\int|W|^{2} d \mu$ is also invariant under the action of the diffeomorphism group, the first variation of $\mathcal{W}$ with respect to any Lie derivative $\dot{g}=\mathcal{L}_{v} g$ must also vanish. Thus

$$
0=\int\left(\nabla^{(a} v^{b)}\right) B_{a b} d \mu=-\int v^{b}\left(\nabla^{a} B_{a b}\right) d \mu
$$

for any vector field $v$, and it follows that we must have

$$
\nabla^{a} B_{a b}=0 .
$$

Thus, the Bach tensor of any metric is automatically divergence-free.

Now the Bianchi identities imply [59] that

$$
\left(\nabla^{a} \nabla^{b}+\frac{1}{2} r^{a b}\right)(\star W)_{c a b d}=0
$$

for any Riemannian 4-manifold, so we can also rewrite the Bach tensor as

$$
B_{a b}=\left(2 \nabla^{c} \nabla^{d}+r^{c d}\right)\left(W_{+}\right)_{a c b d}
$$

where $W_{+}=(W+\star W) / 2$ is the self-dual Weyl curvature. Moreover, since

$$
2 \int_{M}\left|W_{+}\right|^{2} d \mu=\int_{M}|W|^{2} d \mu+12 \pi^{2} \tau(M)
$$

for any Riemannian metric [7, 12, it follows that the vanishing of the Bach tensor $B$ is equivalent to $g$ being a critical point of the functional

$$
\mathcal{W}_{+}(g)=\int\left|W_{+}\right|_{g}^{2} d \mu_{g}
$$

But for a Kähler surface $\left(M^{4}, g, J\right)$ with Kähler form $\omega$,

$$
\left(W_{+}\right)_{a b}{ }^{c d}=\frac{s}{12}\left[\omega_{a b} \omega^{c d}-\delta_{a}^{[c} \delta_{b}^{d]}+J_{a}^{[c} J_{b}^{d]}\right]
$$

so in this case we obtain

$$
B_{a b}=\frac{s}{12} \stackrel{\circ}{a b}_{a b}+\frac{1}{4} J_{a}^{c} J_{b}^{d} \nabla_{c} \nabla_{d} s-\frac{1}{12} \nabla_{a} \nabla_{b} s+\frac{1}{6} g_{a b} \Delta s .
$$

If $g$ happens to be extremal, $\nabla \nabla s$ is $J$-invariant, and this simplifies to become

$$
B=\frac{1}{12}\left[s \stackrel{\circ}{r}+2 \operatorname{Hess}_{0}(s)\right]
$$

where $\operatorname{Hess}_{0}$ denotes the trace-free part of the Hessian $\nabla \nabla$. Moreover, the $J$ invariance of $\nabla \nabla s$ implies that

$$
(\nabla \nabla s)(J \cdot, \cdot)=i \partial \bar{\partial} s
$$


so we deduce the following:

Lemma 6. For any extremal Kähler surface $\left(M^{4}, g, J\right)$, the Bach tensor $B$ of $g$ can be written as $B=\psi(\cdot, J \cdot)$, where $\psi$ is a harmonic $(1,1)$-form. Moreover, $\psi$ is given explicitly by

$$
\psi:=B(J \cdot, \cdot)=\frac{1}{12}[s \rho+2 i \partial \bar{\partial} s]_{0}
$$

where $\rho$ is the Ricci form of $(M, g, J)$ and $[\cdot]_{0}$ denotes projection to the primitive part of a $(1,1)$-form.

Proof. It only remains to show that $\psi$ is harmonic. Since $B$ is divergence-free,

$$
(\delta \psi)_{b}=-\nabla^{a} \psi_{a b}=\nabla^{a} \psi_{b a}=\nabla^{a} B_{c a} J_{b}{ }^{c}=J_{b}{ }^{c} \nabla^{a} B_{a c}=0,
$$

and the $(1,1)$-form $\psi$ is therefore co-closed. But since $B$ is orthogonal to $g, \psi \in \Lambda^{1,1}$ is orthogonal to the Kähler form $\omega$ and so belongs to the primitive $(1,1)$-forms $\Lambda_{0}^{1,1} \subset \Lambda^{1,1}$. However, $\Lambda_{0}^{1,1}=\Lambda^{-}$on a Hermitian manifold of real dimension 4 , so this shows that $\psi$ is an anti-self-dual 2-form. Thus

$$
d \psi=d(-\star \psi)=\star \delta \psi=0,
$$

and the $(1,1)$-form $\psi$ is therefore harmonic, as claimed.

Now consider the Calabi functional

$$
\mathcal{C}(g)=\int s^{2} d \mu
$$

on the space of Kähler metrics. If we restrict this functional to any particular Kähler class, the critical points are by definition 15 just the extremal Kähler metrics. However, if we instead have a critical point of this functional on the space of all Kähler metrics, a critical point must not only be extremal, but must actually be conformally Einstein on the set where $s \neq 0$ :

Proposition 7. Suppose that $g$ is an extremal Kähler metric on a compact complex surface, and suppose that its Kähler class is a critical point of $\mathcal{A}([\omega])$, considered as a function on the Kähler cone. Then $g$ has vanishing Bach tensor. Moreover, on the open subset of $M$ where the scalar curvature $s$ of $g$ is non-zero, the conformally related metric $h=s^{-2} g$ is Einstein.

Proof. Our hypothesis is equivalent to the assumption that $g$ is a critical point of $\mathcal{C}$, considered as a function on the space of all Kähler metrics. But for any Kähler metric in real dimension 4 ,

$$
\left|W_{+}\right|^{2}=\frac{s^{2}}{24}
$$

so this happens iff $g$ is a critical point of the restriction of $\mathcal{W}_{+}$to the space of Kähler metrics. In other words, our hypothesis is true if and only if $g$ is a Kähler metric such that

$$
\int \dot{g}^{a b} B_{a b} d \mu=0
$$

for every $\dot{g}$ arising from a variation through Kähler metrics. But Lemma 6] says that one may find such a variation, with $\dot{\omega}=\psi$, by setting $\dot{g}=B$. It therefore follows that

$$
0=\int \dot{g}^{a b} B_{a b} d \mu=\int|B|^{2} d \mu
$$

and we must therefore have $B \equiv 0$. 
Now recall that if $\hat{g}=u^{2} g$ is any conformal rescaling of a given Riemannian metric $g$, the trace-free Ricci curvature of $\hat{g}$ is given by

$$
\hat{\grave{r}}=\stackrel{\circ}{r}+(n-2) u \operatorname{Hess}_{0}\left(u^{-1}\right),
$$

where $n$ is the real dimension, so that $n=4$ in the case at hand. But we have just shown that $B=0$, so (2) tells us that

$$
\stackrel{\circ}{r}=-2 s^{-1} \operatorname{Hess}_{0}(s) .
$$

Setting $u=s^{-1}$, we therefore conclude that

$$
\hat{\hat{r}}=-2 s^{-1} \operatorname{Hess}_{0}(s)+(4-2) s^{-1} \operatorname{Hess}_{0}(s)=0,
$$

so the conformally related metric $h=s^{-2} g$ is indeed Einstein on the open set $\{p \in M \mid s(p) \neq 0\}$ where it is defined.

The reader should note that Proposition 7 has previously been pointed out by Simanca [63], but, because of the central rôle it plays in the present work, we have thought it important to include a self-contained and transparent proof in this article. The fact that Bach-flat Kähler metrics can be rescaled by their scalar curvatures to yield Einstein metrics has of course been known for much longer and is due to Derdziński [22].

Corollary 8. Let $x_{0}$ be the positive real number of Lemma 3. If the Kähler class $\left(1+x_{0}\right)\left(F_{1}+F_{2}\right)-x_{0} E$ on $M=\mathbb{C P}_{2} \# 2 \overline{\mathbb{C P}}_{2}$ contains an extremal Kähler metric $g$, then $h=s^{-2} g$ is an Einstein metric on $M$.

Proof. The functional $\mathcal{A}([\omega])$ is invariant under rescalings $[\omega] \rightarrow \lambda[\omega]$ and bilateral symmetries $F_{1} \leftrightarrow F_{2}$. Thus any critical point of the restriction of $\mathcal{A}$ to the classes of the form $(1+x)\left(F_{1}+F_{2}\right)-x E$ is also a critical point of $\mathcal{A}$ itself. But since

$$
f(x)=\mathcal{A}\left((1+x)\left(F_{1}+F_{2}\right)-x E\right),
$$

Lemma 3 exactly tells us that $\left(1+x_{0}\right)\left(F_{1}+F_{2}\right)-x_{0} E$ is such a critical point. Since Lemma 4 also tells us that such an extremal Kähler metric would automatically have $s>0$, Proposition 7 then guarantees that $h=s^{-2} g$ would be an Einstein metric, defined on all of $M$.

\section{Sobolev CONSTANTS}

If $(M, J)$ is a compact complex surface, we will say that a Kähler class [ $\omega]$ on $M$ belongs to the controlled cone if

$$
c_{1}^{2}(M)-\frac{2}{3}\left[\frac{\left(c_{1} \cdot[\omega]\right)^{2}}{[\omega]^{2}}-\mathcal{F}([\omega], \xi)\right]>0 .
$$

This is equivalent to requiring that

$$
\mathcal{A}([\omega])<\frac{3}{2} c_{1}^{2},
$$

so that an extremal Kähler metric $g$ in $[\omega]$ would then satisfy

$$
\frac{1}{32 \pi^{2}} \int_{M} s^{2} d \mu<\frac{3}{2} c_{1}^{2} .
$$


Now our computations in $\$ 3$ for bilaterally symmetric classes show that

$$
\begin{aligned}
\mathcal{A}([\omega]) & =3\left(\frac{32+176 x+318 x^{2}+280 x^{3}+132 x^{4}+32 x^{5}+3 x^{6}}{12+72 x+138 x^{2}+120 x^{3}+54 x^{4}+12 x^{5}+x^{6}}\right) \\
& =9\left(\frac{32+176 x+318 x^{2}+280 x^{3}+132 x^{4}+32 x^{5}+3 x^{6}}{36+216 x+414 x^{2}+360 x^{3}+162 x^{4}+36 x^{5}+3 x^{6}}\right) \\
& <9,
\end{aligned}
$$

as follows by term-by-term comparison of the numerator and denominator. Since $c_{1}^{2}\left(\mathbb{C P}_{2} \# 2 \overline{\mathbb{C P}}_{2}\right)=7$, we thus have

$$
\mathcal{A}([\omega])<9<10.5=\frac{3}{2} c_{1}^{2}(M)
$$

for any bilaterally symmetric class. This shows the following:

Lemma 9. Any bilaterally symmetric Kähler class $[\omega]$ on $M=\mathbb{C P}_{2} \# 2 \overline{\mathbb{C P}}_{2}$ belongs to the controlled cone.

By refining an idea first suggested by Gang Tian 67, we will now show that this allows us to prove uniform estimates of the Yamabe constants and Sobolev constants of these metrics; cf. [20, 71]. Let us first recall that the Yamabe constant of a conformal class $[g]$ of Riemannian metrics on a compact 4-manifold $M$ is the number

$$
Y_{[g]}=\inf _{\hat{g} \in[g]} \frac{\int_{M} s_{\hat{g}} d \mu_{\hat{g}}}{\sqrt{\int_{M} d \mu_{\hat{g}}}} .
$$

By the celebrated work of Trudinger, Aubin, and Schoen 9, 46 the infimum for any conformal class $[g]$ is actually achieved by some metric, and this so-called Yamabe minimizer $g_{Y} \in[g]$ necessarily has constant scalar curvature.

Now the scalar curvature of a metric $\hat{g}=u^{2} g$ conformal to $g$ satisfies

$$
s_{\hat{g}} u^{3}=(6 \Delta+s) u,
$$

where $\Delta$ is the positive Laplacian, so the Yamabe constant may be re-expressed as

$$
Y_{[g]}=\inf _{u \neq 0} \frac{\int\left(6|\nabla u|^{2}+s_{g} u^{2}\right) d \mu_{g}}{\left(\int u^{4} d \mu_{g}\right)^{1 / 2}}
$$

and notice that we are now allowing ourselves to consider even those smooth $u$ which change sign, since replacing $u$ with a positive smoothing of $|u|$ would otherwise decrease the quotient on the right. If $Y_{[g]}>0$, we thus have

$$
\|u\|_{L^{4}}^{2} \leq \frac{6}{Y_{[g]}}\|\nabla u\|_{L^{2}}^{2}+\frac{\max s_{g}}{Y_{[g]}}\|u\|_{L^{2}}^{2}
$$

for all $u \in L_{1}^{2}$. In particular, if we define 2, 71] the Sobolev constant $C_{S}$ of $g$ to be the smallest constant such that the estimate

$$
\|u\|_{L^{4}}^{2} \leq C_{S}\left(\|\nabla u\|_{L^{2}}^{2}+V^{-1 / 2}\|u\|_{L^{2}}^{2}\right)
$$

holds, where $V$ is the total volume of $(M, g)$, then we automatically have

$$
C_{S} \leq \frac{\max \left(6, s_{\max } V^{1 / 2}\right)}{Y_{[g]}}
$$

for any compact Riemannian 4-manifold $(M, g)$ with $Y_{[g]}>0$. 
Now the Gauss-Bonnet and signature theorems for a smooth compact oriented 4-manifold $M$ imply that

$$
\frac{1}{4 \pi^{2}} \int_{M}\left(\frac{s^{2}}{24}+2\left|W_{+}\right|^{2}-\frac{|\grave{r}|^{2}}{2}\right) d \mu_{g}=(2 \chi+3 \tau)(M)
$$

for every Riemannian metric on $M$. If $M$ admits an orientation-compatible almostcomplex structure, $(2 \chi+3 \tau)(M)=c_{1}^{2}$, and we therefore have

$$
\int_{M}\left(\frac{s^{2}}{24}+2\left|W_{+}\right|^{2}\right) d \mu_{g} \geq 4 \pi^{2} c_{1}^{2}
$$

However, $\mathcal{W}_{+}=\int\left|W_{+}\right|^{2} d \mu$ is conformally invariant, so, by applying this inequality to a Yamabe minimizer $g_{Y} \in[g]$, we obtain

$$
Y_{[g]}^{2} \geq 96 \pi^{2} c_{1}^{2}-48 \mathcal{W}_{+}(g)
$$

for every Riemannian metric $g$. In the special case when $g$ is Kähler, we have

$$
\mathcal{W}_{+}(g)=\int_{M} \frac{s^{2}}{24} d \mu
$$

so this gives us

$$
Y_{[g]}^{2} \geq 96 \pi^{2} c_{1}^{2}-2 \int_{M} s^{2} d \mu
$$

in the Kähler case. When $g$ is extremal,

$$
\int_{M} s^{2} d \mu=32 \pi^{2} \mathcal{A}([\omega])
$$

and we therefore conclude that

$$
Y_{[g]}^{2} \geq 64 \pi^{2}\left(\frac{3}{2} c_{1}^{2}-\mathcal{A}([\omega])\right) .
$$

Thus Yamabe constants are bounded away from zero in the interior of the controlled cone.

Now Lemma 4 tells us that any bilaterally symmetric extremal Kähler metric $g$ on $M=\mathbb{C P}_{2} \# 2 \overline{\mathbb{C P}}_{2}$ has positive scalar curvature and hence has positive Yamabe constant $Y_{[g]}$. Since $c_{1}^{2}(M)=7$ and $\mathcal{A}(g)<9$, inequality (4) therefore tells us that these metrics all satisfy

$$
Y_{[g]}>8 \pi \sqrt{\frac{3}{2}(7)-9}=4 \pi \sqrt{6}
$$

With Lemma 5 and inequality (3), this then tells us that the Sobolev constants of these metrics satisfy the uniform bound

$$
C_{S}<\frac{\max (6,24 \pi \sqrt{2})}{4 \pi \sqrt{6}}=2 \sqrt{3} .
$$

But, by previous work of the first and third authors [20], a uniform upper bound on Sobolev constants implies a weak compactness statement:

Theorem 10. Let $g_{i}$ be an arbitrary sequence of unit-volume bilaterally symmetric extremal Kähler metrics on $M=\mathbb{C P}_{2} \# 2 \overline{\mathbb{C P}}_{2}$. Then there is a subsequence $g_{i_{3}}$ of these metrics which converges in the Gromov-Hausdorff topology to an extremal Kähler metric on a compact complex 2-orbifold. 
Now in general, the limit orbifold can certainly be different from $M$; in particular, the construction of Arezzo, Pacard, and Singer 4 s shows that when $x \rightarrow 0$, the limit orbifold is the manifold $\mathbb{C P}_{1} \times \mathbb{C P}_{1}$, whereas when $x \rightarrow \infty$, the limit orbifold is the manifold $\mathbb{C P}_{2}$. In order to prove Theorem $\mathrm{A}$, what we therefore need to do is rule out the bubbling off of curvature and topology in the case of increasing sequences of $x \in\left(0, x_{0}\right]$.

\section{Formation of Bubbles}

Without further work, the results of 20] only allow us to conclude that sequences of extremal metrics with bounded Sobolev constant have orbifold limits in the Gromov-Hausdorff sense. However, just as in the earlier work of Anderson [1, 2, 3] and of Tian and Viaclovsky [69, 71], the orbifold singularities can only arise by a very specific mechanism of curvature concentration.

Suppose we have a sequence of unit-volume extremal Kähler metrics $g_{i}$, and let us also assume that we have upper and lower bounds of their scalar curvatures:

$$
\left|s_{g_{i}}\right|<\text { const. }
$$

Assuming the uniform Sobolev constant bounds, the curvatures of these metrics becomes unboundedly large at a point only if the $L^{2}$ norm of curvature reaches a definite threshold on arbitrarily small balls, in the precise sense that there are universal constants $C, \epsilon_{0}>0$ (depending only on the dimension and the Sobolev constant) so that, if we set $\varrho=C\left|\mathcal{R}_{p}\right|^{-2}$, we then have

$$
\int_{B_{\varrho}(p)}|\mathcal{R}|^{2} d \mu \geq \epsilon_{0}
$$

If there is no uniform bound for the sectional curvatures of the $g_{i}$, we can choose points $p_{i}$ centered at points of large curvature, rescale so that $\left|\mathcal{R}_{p_{i}}\right|=1$, and then take a pointed limit of some subsequence. The limit (which is called a bubble) will then be a complete extremal Kähler orbifold of total energy $\int|\mathcal{R}|^{2} d \mu \geq \epsilon_{0}$. Because the metric was rescaled at each stage by factors tending toward infinity, the scalar curvatures are commensurately multiplied by factors tending toward zero, and our assumed uniform bounds on $s$ then imply that the limit orbifold is actually scalar-flat Kähler.

The bubble's structure at infinity is also known. The Sobolev constant bound implies a global Euclidean volume growth lower bound on the orbifold. The $\epsilon_{0^{-}}$ regularity theorem for extremal metrics asserts that

$$
\sup _{B_{\varrho / 2}(p)}|\mathcal{R}| \leq C \varrho^{-2}\left(\int_{B(\varrho, p)}|\mathcal{R}|^{2}\right)^{1 / 2} \text { whenever } \int_{B_{\varrho}(p)}|\mathcal{R}|^{2} \leq \epsilon_{0} .
$$

Since the total energy is bounded, points far enough away from the basepoint must be at the center of large balls of small energy and so that $|\mathcal{R}|=o\left(\mathrm{~s}^{-2}\right)$, where я denotes the Euclidean radius in asymptotic coordinates; moreover, since the bubbles that actually concern us here will actually be anti-self-dual 4-manifolds, we can appeal to the results of Tian and Viaclovsky [70, Proposition 5.2] to obtain the faster curvature fall-off $|\mathcal{R}|=o\left(\mathrm{~s}^{-4+\delta}\right)$, for any $\delta>0$, at infinity. Results of Anderson [2] and of Tian and Viaclovski [71] also show that the bubble has only finitely many ends, each of which is asymptotically locally Euclidean (ALE), meaning each end is asymptotic to the standard cone metric on $S^{3} / \Gamma$. In our case 
one can improve this even further, as results of $\mathrm{Li}$ and Tam [48, Theorem 4.1], 49, Theorem 1.9] then imply that an ALE Kähler manifold has just one end 2

Now even these rescaled metrics may have curvature concentration points; however, we can then repeat the above procedure by picking points $q_{i}$ a finite distance from $p_{i}$ that have $\left|\mathcal{R}_{q_{i}}\right| \rightarrow \infty$ and rescaling and taking another pointed limit. The above-cited regularity theorem from [20] implies that each stage must have a ball of large radius that, after removing the points of curvature concentration, has at least $\epsilon_{0}$ worth of energy. Since there is a finite amount of total energy available, this process must eventually terminate with a single-ended, scalar-flat ALE Kähler manifold. Any blow-up limit that yields a smooth metric will be called a deepest bubble. Obviously, no curvature can bubble off at all unless such a deepest bubble can be constructed. Indeed, the absence of a deepest bubble would imply a uniform bound on sectional curvature, leading to smooth convergence everywhere.

Let us now focus on the special case of a sequence $g_{i}$ of unit-volume bilaterally symmetric extremal Kähler metrics on $M=\mathbb{C P}_{2} \# 2 \overline{\mathbb{C P}}_{2}$. First notice that Lemma 5 asserts that such metrics do in fact satisfy a uniform scalar curvature bound, so the above discussion does indeed apply. Since the curvature of any deepest bubble $\left(X, g_{\infty}\right)$ necessarily arises from a concentration of the curvatures of the $g_{i}$, we thus have the following:

Lemma 11. Let $g_{i}$ be a sequence of metrics as in Theorem 10. If $g_{i}$ fails to converge modulo diffeomorphisms in the smooth topology, then there is a non-trivial asymptotically locally Euclidean (ALE) scalar-flat Kähler manifold $\left(X, g_{\infty}\right)$ which arises as a pointed Gromov-Hausdorff limit of rescalings of a subsequence of the $g_{i}$. Moreover, the trace-free Ricci curvature and anti-self-dual Weyl curvature $W_{+}$of $X$ necessarily satisfy

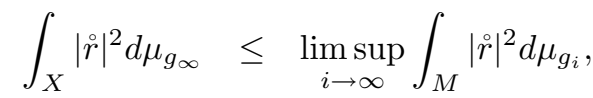

$$
\begin{aligned}
& \int_{X}\left|W_{-}\right|^{2} d \mu_{g_{\infty}} \leq \limsup _{i \rightarrow \infty} \int_{M}\left|W_{-}\right|^{2} d \mu_{g_{i}} .
\end{aligned}
$$

Because [40] scalar-flat Kähler surfaces are anti-self-dual as oriented Riemannian 4-manifolds, the following regularity observation therefore applies to the present context.

Proposition 12. Let $\left(X, g_{\infty}\right)$ be any ALE anti-self-dual 4-manifold, where

$$
g_{\infty}=\text { Euclidean }+o\left(\mathrm{~s}^{-2+\delta}\right), \partial g_{\infty}=o\left(\mathrm{~s}^{-3+\delta}\right),
$$

for some $\delta \in(0,1 / 2)$, where a denotes the Euclidean radius. Consider the orbifold compactification $\hat{X}$ of $X$ obtained by adding an extra point at each end of $X$. Then $\hat{X}$ carries a canonical real-analytic structure such that the conformal class $\left[g_{\infty}\right]$ extends to $\hat{X}$ as a real-analytic anti-self-dual conformal metric $[\hat{g}]$.

\footnotetext{
${ }^{2}$ In real dimension 4 , this assertion can be seen more directly by first showing that such a manifold can be compactified into a compact complex surface by adding a divisor of positive selfintersection at each end. But for any compact complex surface, the intersection form on $H^{1,1}$ is always [1] either Lorentzian or negative definite, so any two divisors of positive self-intersection must necessarily meet. The existence of two or more ends would thus lead to an immediate contradiction.
} 


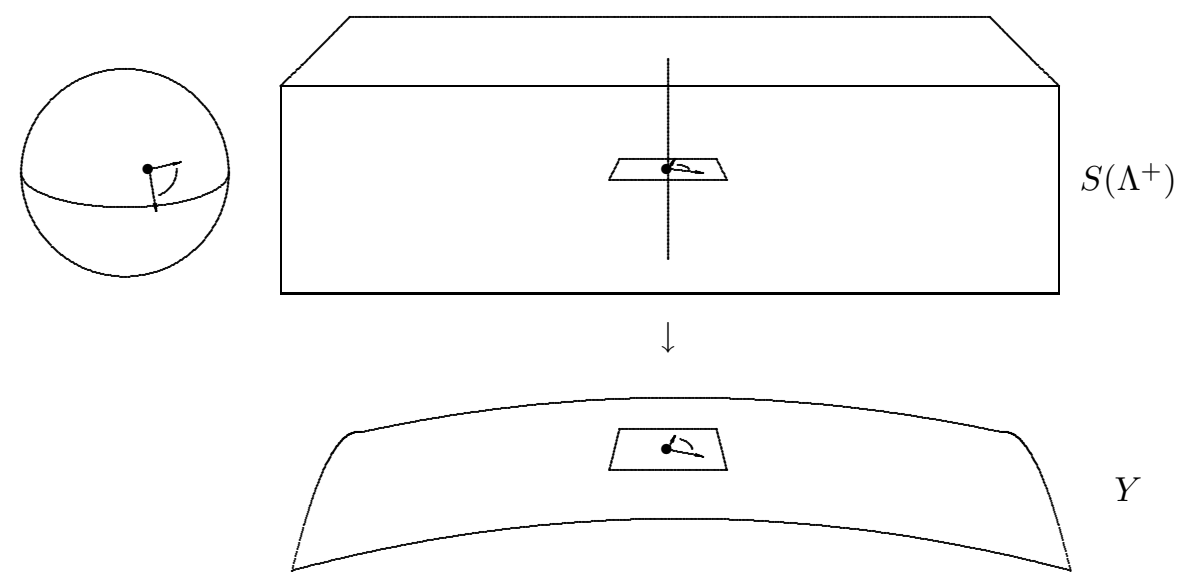

FiguRE 4

Proof. In inverted coordinates, the conformally related metric $\hat{g}=g_{\infty} \mathrm{A}^{-4}$ is Euclidean $+o\left(\varrho^{2-\delta}\right)$, where $\varrho=1 /$ s, so $g_{\infty}$ thus determines a $C^{1, \alpha}$ conformal metric $\hat{g}_{0}$ on the orbifold compactification $\hat{X}$ of $X$, for any $\alpha \in(1 / 2,1-\delta)$. Let $Y \approx \mathbb{R}^{4}$ be a uniformizing chart for any end of $X$. The Christoffel symbols of $\hat{g}_{0}$ are thus of class $C^{0, \alpha}$ and the standard Atiyah-Hitchin-Singer formulation of the twistor construction [7, 40] (see Figure 4) gives us an almost-complex structure $J$ on the 6 -manifold $Z$ defined as the 2 -sphere bundle $S\left(\Lambda^{+}\right) \rightarrow Y$. We now apply the HillTaylor version [29] of the Newlander-Nirenberg theorem for rough almost-complex structures. Since $J$ is an almost-complex structure of class $C^{0, \alpha}, \alpha>1 / 2$, its Nijenhuis tensor $\mathcal{N}_{J}$ is not only well-defined in the distributional sense, but actually [29. Lemma 1.2, Remark 1.5] of Sobolev regularity better than $L_{-1 / 2}^{2}$. But since $\left[\hat{g}_{0}\right]=\left[g_{\infty}\right]$ is anti-self-dual away from the added point at infinity, $\mathcal{N}_{J}$ is supported at the added twistor fiber, which is a submanifold of $Z$. However, a distribution of Sobolev class $L_{-1 / 2}^{2}$ that is supported on a real hypersurface is automatically zero, as follows from [50, Chapter 1, Theorem 11.1] and duality. Thus it follows that $\mathcal{N}_{J}=0$ in the distributional sense, and $(Z, J)$ is therefore [29, Theorem 1.1] a complex manifold. Inverting the twistor correspondence [32, 43, 58, therefore realizes $Y$ as the real slice of the moduli space of rational curves $\mathbb{C P}_{1} \subset Z$ with normal bundle $\mathcal{O}(1) \oplus \mathcal{O}(1)$; and, more importantly, this moduli space carries an anti-holomorphic involution fixing $Y$ and a natural holomorphic conformal structure whose restriction to $Y$ is $\left[\hat{g}_{0}\right]$. In particular, $Y$ can be given a real-analytic structure in which $\left[\hat{g}_{0}\right]$ is represented by a real-analytic metric $\hat{g}$. Dividing $Y$ by the appropriate group $\Gamma$ now gives us an orbifold chart associated with the given end, and repeating the same argument for each end then gives $\hat{X}$ the promised structure.

Notice that the results of Tian and Viaclovsky [70 tell us that the hypotheses of this proposition hold whenever an anti-self-dual manifold $\left(X, g_{\infty}\right)$ arises as a bubble. This has many useful consequences:

Proposition 13. Let $\left(X, g_{\infty}\right)$ be any scalar-flat ALE anti-self-dual 4-manifold, where

$$
g_{\infty}=\text { Euclidean }+o\left(\mathrm{~s}^{-2+\delta}\right), \partial g_{\infty}=o\left(\mathrm{~s}^{-3+\delta}\right),
$$


for some $\delta \in(0,1 / 2)$, where $\mathrm{g}$ denotes the Euclidean radius. Then $X$ has negative intersection form. Moreover, after possibly passing to better charts at infinity, $g_{\infty}$ actually satisfies the improved fall-off conditions

$$
g_{\infty}=\text { Euclidean }+O\left(\mathrm{~s}^{-2}\right), \partial^{m} g_{\infty}=O\left(\mathrm{~s}^{-2-m}\right) \forall m \geq 1 .
$$

In particular, these conclusions apply to any deepest bubble $\left(X, g_{\infty}\right)$ arising as in Lemma 11.

Proof. The real-analytic conformal class $[\hat{g}]$ can be represented by a real-analytic metric $\hat{g}$ whose scalar-curvature $s$ does not change sign; for example, such a metric can be constructed via Trudinger's trick of rescaling by the lowest eigenfunction of the Yamabe Laplacian. Setting $g_{\infty}=u^{2} \hat{g}$ for $u>0$, one then finds that $u$ is real-analytic and proper on $\hat{X}-X$ and solves the equation

$$
0=\left(\Delta+\frac{s}{6}\right) u,
$$

so that we must have $s>0$ by examination of the minima of $u$.

Let us now represent the deRham groups $H^{2}(\hat{X})$ by harmonic 2-forms with respect to $\hat{g}$. Letting $\varphi$ be any such harmonic 2-form, its self-dual part $\varphi^{+}=$ $(\varphi+\star \varphi) / 2$ then satisfies the Weitzenböck formula

$$
0=\left(d+d^{*}\right)^{2} \varphi^{+}=\nabla^{*} \nabla \varphi^{+}-2 W\left(\varphi^{+}, \cdot\right)+\frac{s}{3} \varphi^{+}=\nabla^{*} \nabla \varphi^{+}+\frac{s}{3} \varphi^{+} .
$$

Taking the inner product with $\varphi^{+}$and integrating, we thus conclude that $\varphi^{+}$vanishes. Hence any harmonic form on $\hat{X}$ is anti-self-dual, and the intersection form of $\hat{X}$ is negative. Since $H^{2}(X)=H_{c}^{2}(X)=H^{2}(\hat{X})$, the same therefore applies to our original manifold $X$.

Now (6) reveals that $u$ is in fact a linear superposition of the Yamabe Green's functions of the ends. Since the Yamabe Green's function of an anti-self-dual manifold is real-analytic in any real-analytic conformal gauge and has a local expansion [5]

$$
G_{y}=\frac{1}{4 \pi^{2} \varrho^{2}}+\text { bounded }
$$

without $\log (\varrho)$ term, the improved asymptotic expansion for $g_{\infty}$ now follows by inverting geodesic coordinates about each point of $\hat{X}-X$.

This said, we now immediately have the following:

Lemma 14. Let $g_{i}$ and $\left(X, g_{\infty}\right)$ be as in Lemma 11, Then $X$ is diffeomorphic to an open subset of $M$. Moreover, $b_{1}(X)=b_{3}(X)=0$, and $b_{2}(X) \leq 2$.

Proof. The bubble $\left(X, g_{\infty}\right)$ is obtained as a pointed Gromov-Hausdorff limit of rescaled versions of small metric balls in $M$, and the rescaling is done in such a manner as to arrange that the sectional curvatures are bounded. One therefore gets smooth convergence on compact subsets by passing to a subsequence and applying suitable diffeomorphisms. But $X$ is diffeomorphic to the interior of a compact domain $U \subset X$ with smooth boundary $S^{3} / \Gamma$. This domain can then be mapped diffeomorphically into the manifold, resulting in a decomposition

$$
M \approx U \cup_{S^{3} / \Gamma} V
$$


where $U$ and $V$ are manifolds-with-boundary, $X \approx \operatorname{Int}(U)$, and $\partial U=\partial V=S^{3} / \Gamma$. Since $M=\mathbb{C P}_{2} \# 2 \overline{\mathbb{C P}}_{2}$ is simply connected, the Mayer-Vietoris sequence tells us that both $U$ and $V$ have $b_{1}=b_{3}=0$, while

$$
H^{2}(M, \mathbb{R})=H^{2}(U, \mathbb{R}) \oplus H^{2}(V, \mathbb{R}) .
$$

Since the analogous statements similarly hold for homology, the intersection form of $X \approx \operatorname{Int}(U)$ is just the restriction of the intersection form of $H^{2}(M)$ to the linear subspace $H^{2}(U) \subset H^{2}(M)$. But the intersection form of $X$ is negative by Proposition 13, and it thus follows that $b_{2}(X) \leq b_{-}(M)=2$.

The following will also prove quite useful.

Lemma 15. Let $g_{i}$ and $\left(X, g_{\infty}\right)$ be as in Lemma 11. If the open subset of Lemma 14 cannot be taken to be invariant under $F_{1} \leftrightarrow F_{2}$, then curvature is accumulating in more than one region and

$$
\begin{aligned}
2 \int_{X}|\grave{r}|^{2} d \mu_{g_{\infty}} & \leq \limsup _{i \rightarrow \infty} \int_{M}|\grave{r}|^{2} d \mu_{g_{i}}, \\
2 \int_{X}\left|W_{-}\right|^{2} d \mu_{g_{\infty}} & \leq \limsup _{i \rightarrow \infty} \int_{M}\left|W_{-}\right|^{2} d \mu_{g_{i}} .
\end{aligned}
$$

Proof. If we go far out in the sequence, the deepest bubble essentially arises by rescaling the interior of a domain $U_{j}$ of small diameter, where the $L^{2}$ norms of curvatures on $U_{j}$ closely approximate the corresponding norms for the deepest bubble $X$. Now move this domain by the isometry $F_{1} \leftrightarrow F_{2}$ to obtain another domain $U_{j}^{\prime}$. If, after again passing to a subsequence, the rescaled distance from $U_{j}$ to $U_{j}^{\prime}$ remains bounded, the pointed Gromov-Hausdorff limit will include the limits of the $U_{j}^{\prime}$, and we will have an induced isometry which exchanges the two. Otherwise, the concentration of curvature represented by the $U_{j} \cup U_{j}^{\prime}$ is reflected not by $\left(X, g_{\infty}\right)$, but rather by two disjoint copies of it, and we therefore get a factor of two in the relevant curvature inequalities.

Proposition 16. Let $\left(X, g_{\infty}\right)$ be a deepest bubble, as in Lemma 11, Then $g_{\infty}$ is toric, and $H_{2}(X)$ is generated by holomorphically embedded $\mathbb{C P}_{1}$ 's in $X$.

Proof. Recall that a Killing vector field $\eta$ on a Riemannian manifold is completely determined by its 1-jet at any point $p$, since the restriction of such a field to any geodesic solves a second-order ODE - namely, Jacobi's equation. Because Killing's equation says $\nabla_{a} \eta_{b}=-\nabla_{b} \eta_{a}$, the initial data for a Killing field may be identified with $\Lambda_{p}^{1} \oplus \Lambda_{p}^{2}$. If we equip the bundle $\Lambda^{1} \oplus \Lambda^{2}$ with a connection defined by

$$
D_{a}\left(\eta_{b}, \varphi_{c d}\right):=\left(\nabla_{a} \eta_{b}-\varphi_{a b}, \nabla_{a} \varphi_{c d}-\mathcal{R}_{a c d}^{e} \eta_{e}\right),
$$

we thus conclude that Killing fields precisely correspond, via $\eta^{a} \mapsto\left(\eta_{b}, \nabla_{c} \eta_{d}\right)$, to $D$-parallel sections of $\Lambda^{1} \oplus \Lambda^{2}$. A constant rescaling $g \rightsquigarrow c g$ of the metric merely induces a homothety $(\eta, \varphi) \mapsto(c \eta, c \varphi)$ and so in particular does not affect the correspondence between 1-parameter subgroups of the isometry group and parallel line-sub-bundles of $\Lambda^{1} \oplus \Lambda^{2}$.

Since $\left(X, g_{\infty}\right)$ is constructed as a pointed limit, it comes equipped with a basepoint $p \in X$ which, after passing to a suitable subsequence, can be thought of as the limit of a sequence of points $p_{i} \in M$ associated with rescalings $c_{i} g_{i}$ of the given metrics. Since each of the metrics $g_{i}$ is toric, the generators of the torus action span a 2-plane $\Pi_{i} \subset \Lambda_{p_{i}}^{1} \oplus \Lambda_{p_{i}}^{2}$, and since, by construction, $T_{p} X$ is canonically 
identified with each $T_{p_{i}} M$, we therefore obtain a sequence in the Grassmannian $G r_{2}\left(\Lambda_{p}^{1} \oplus \Lambda_{p}^{2}\right)$. But the latter is compact, so we may arrange, by again passing to a subsequence, that $\Pi_{i} \rightarrow \Pi$ for some 2 -plane $\Pi \subset \Lambda_{p}^{1} \oplus \Lambda_{p}^{2}$. Since the pull-backs of the rescaled metrics $c_{i} g_{i}$ via suitable diffeomorphisms converge in $C^{\infty}$ to $g_{\infty}$ on compact subsets of $X$, the holonomy transformation of $\Lambda_{p}^{1} \oplus \Lambda_{p}^{2}$ induced by $D$ parallel transport in $\Lambda^{1} \oplus \Lambda^{2} \rightarrow X$ around any smooth loop in $\gamma$ based at $p$ is always the limit of the holonomy transformations in $\left(M, c_{i} g_{i}\right)$ around loops based at $p_{i}$, and since the latter holonomy transformations act trivially on $\Pi_{i}$, it follows that $\Pi \subset \Lambda_{p}^{1} \oplus \Lambda_{p}^{2}$ is invariant under the holonomy transformations induced by $D$-parallel transport. Hence $\Pi$ extends uniquely via $D$-parallel transport to a $D$-parallel subbundle of $\Lambda^{1} \oplus \Lambda^{2} \rightarrow X$, and we thereby obtain two non-proportional Killing fields on $\left(X, g_{\infty}\right)$. Moreover, these Killing fields can now be seen to arise, under suitable diffeomorphisms, as smooth limits on compact sets of linear combinations of the original two commuting Killing fields, so the two Killing fields $\eta, \tilde{\eta}$ we obtain in this way on $X$ automatically commute with each other.

Now the two Killing fields $\eta$ and $\tilde{\eta}$ become conformal Killing fields on the antiself-dual orbifold $\hat{X}=X \cup\{\infty\}$ which vanish at the added orbifold point. But Pontecorvo 60] has pointed out that, even locally, any conformally flat scalar-flat Kähler surface is locally symmetric, and it therefore follows that a non-flat ALE scalar-flat Kähler surface like $\left(X, g_{\infty}\right)$ can never be conformally flat. The compact orbifold $\hat{X}=X \cup\{\infty\}$ therefore has $W_{-} \not \equiv 0$. Thus the usual proofs of the FerrandLelong/Obata theorem [47, 55] apply in this orbifold setting, and the conformal group of $(\hat{X},[\hat{g}])$ coincides with the isometry group of some orbifold metric in the conformal class. Since our conformal Killing fields $\eta$ and $\hat{\eta}$ fix the orbifold point $\infty \in \hat{X}$, their action on $\hat{X}$ is therefore completely determined (via the exponential map) by their action on the tangent space at $\infty$ in a local uniformizing chart. Up to finite covers, this therefore gives us a faithful $S O(4)$-valued representation of the group generated by $\eta$ and $\tilde{\eta}$. But $\eta$ and $\tilde{\eta}$ are independent, and $[\eta, \tilde{\eta}]=0$. The 2-dimensional Abelian Lie group they generate must therefore be covered by a maximal torus in $\mathrm{SO}(4)$ and so must be compact. Hence the Killing fields $\eta$ and $\hat{\eta}$ generate an action of the compact group $T^{2}$ on $\left(X, g_{\infty}\right)$. Moreover, by replacing $\eta$ and $\hat{\eta}$ with linear combinations, we may find an asymptotic chart for $X$ in which

$$
\begin{aligned}
\eta & =x^{1} \frac{\partial}{\partial x^{2}}-x^{2} \frac{\partial}{\partial x^{1}}+O(1) \\
\tilde{\eta} & =x^{3} \frac{\partial}{\partial x^{4}}-x^{4} \frac{\partial}{\partial x^{3}}+O(1) \\
g_{\infty} & =\text { Euclidean }+O\left(\mathrm{~s}^{-2}\right), \\
\partial g_{\infty} & =O\left(\mathrm{~s}^{-3}\right) .
\end{aligned}
$$

Now the original Killing fields on $\left(M, c_{i} g_{i}\right)$ were real-holomorphic, so their 1-jets in fact all belonged to $\Lambda_{p_{i}}^{1} \oplus \Lambda_{p_{i}}^{1,1}$. It follows that the limit plane $\Pi$ is therefore a sub-space of $\Lambda_{p}^{1} \oplus \Lambda_{p}^{1,1}$, and $\eta$ and $\tilde{\eta}$ are therefore also real holomorphic. Since these fields preserve both the metric $g_{\infty}$ and the limit complex structure $J=J_{\infty}$, they therefore preserve the limit Kähler form $\omega=\omega_{\infty}$, too. We can thus arrange that in our asymptotic chart we also have

$$
\omega=d x^{1} \wedge d x^{2}+d x^{3} \wedge d x^{4}+O\left(s^{-2}\right) .
$$


By Lemma 14 $b_{1}(X)=0$, so both $\eta$ and $\tilde{\eta}$ are globally Hamiltonian; that is, there exist smooth functions $t_{1}, t_{2}: X \rightarrow \mathbb{R}$ such that

$$
\omega(\eta, \cdot)=-d t_{1}, \omega(\tilde{\eta}, \cdot)=-d t_{2},
$$

and the above asymptotics therefore give us

$$
\begin{aligned}
& t_{1}=\frac{\left|x^{1}\right|^{2}+\left|x^{2}\right|^{2}}{2}+O(\text { я), } \\
& t_{2}=\frac{\left|x^{3}\right|^{2}+\left|x^{4}\right|^{2}}{2}+O(\text { я). }
\end{aligned}
$$

This shows that $t_{1}+t_{2}>\mathrm{s}^{2} / 3$ on the complement of a compact set, and it therefore follows that the moment map

$$
\vec{t}=\left(t_{1}, t_{2}\right): X \longrightarrow \mathbb{R}^{2}
$$

is proper. Moreover, any linear combination $a_{1} t_{1}+a_{2} t_{2}$, where $a_{1}$ and $a_{2}$ are positive constants, is proper by the same argument. Since $\eta$ and $\tilde{\eta}$ are Killing fields on our Kähler manifold, a generic such linear combination has only non-degenerate critical points and is therefore a Morse function. The essence of [ 6 ] therefore applies, despite our non-compact setting. Namely, the image of $\vec{t}$ is a convex subset of the plane, bounded by two half-lines and a finite number of line segments of rational slope:

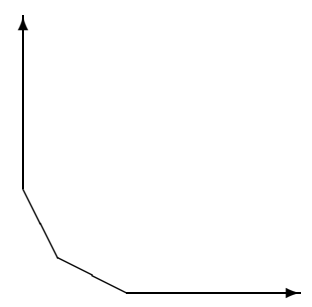

The two boundary rays arise from the fixed-point sets of $\eta$ and $\tilde{\eta}$, which are totally geodesic copies of $\mathbb{C}$ emanating from two fixed points of the torus action. The boundary segments arise from other sets where some circle subgroup of $T^{2}$ acts trivially. The inverse image of each such segment is a totally geodesic surface and must be a topological 2 -sphere because it carries an $S^{1}$-action with two fixed points; moreover, each such 2-sphere is the zero locus of a holomorphic vector field and so is a holomorphic curve. Finally, the union of these $\mathbb{C P}_{1}$ 's is a deformation retraction of $X$, as may be accomplished by pushing along the flow of some Morse function $a_{1} t_{1}+a_{2} t_{2}$. Thus $b_{2}(X)$ is exactly the number of line segments, and $H_{2}(X)$ is generated by holomorphically embedded $\mathbb{C P}_{1}$ 's, as promised.

Given the amount of structure we have already displayed, it seems extremely plausible that our toric manifolds $\left(X, g_{\infty}\right)$ actually number among the scalar-flat Kähler instantons explicitly constructed by Calderbank and Singer [17. The latter arise via a special form of the Joyce ansatz, and one of Joyce's results 34, Theorem 2.4.5] in any case implies that our metrics $g_{\infty}$ are at least locally expressible in his framework. Moreover, the results of Fujiki [26], although not immediately applicable here, make it seem very likely that a global result along these lines should actually hold. 


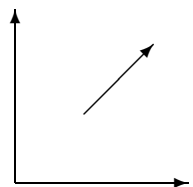

Figure 5

For our purposes, however, it will not actually be necessary to know the possible bubble metrics $g_{\infty}$ in closed form. Instead, the next few lemmas will supply all the information we need.

Lemma 17. Let $X$ be as in Lemma 11. Then $b_{2}(X) \neq 0$.

Proof. If $b_{2}(X)=0$, the proof of Proposition 16 shows that there is a Morse function on $X$ with exactly one critical point (see Figure 5 ). Thus $X$ is diffeomorphic to $\mathbb{R}^{4}$. Hence $\hat{X}=X \cup\{\infty\}$ is diffeomorphic to $S^{4}$, and the signature formula then shows that $\hat{X}$ is conformally flat. Thus $\left(X, g_{\infty}\right)$ is a conformally flat scalar-flat Kähler manifold, and Pontecorvo's theorem [60 therefore tells us that it is locally symmetric. Its curvature fall-off at infinity therefore forces $\left(X, g_{\infty}\right)$ to be flat 3 . But a deepest bubble $X$ cannot be flat, so this case simply never arises.

While this result may look innocuous, it is actually heavily dependent on the fact that $X$ is known to be toric. For example, there is a non-trivial Ricci-flat ALE metric (with isometry group $S O(3)$ ) on $\mathbb{C P}_{2}$ minus a smooth conic; but this space is diffeomorphic to $T \mathbb{R} \mathbb{P}^{2}$ and so has $b_{2}=0$. Perhaps the most dramatic consequence of the toric condition is that it guarantees the existence of a Morse function whose critical points all have even index; thus $X$ must, in particular, be simply connected. We leave it as an exercise to check that, more generally, any simply connected scalar-flat Kähler bubble must have $b_{2} \neq 0$. The point is that $X$ must then either be hyper-Kähler, in which case one can appeal to the results of Kronheimer [36, or else the Ricci form will correspond to a non-trivial bounded harmonic 2 -form on $\hat{X}$.

Lemma 18. Let $\left(X, g_{\infty}\right)$ be a deepest bubble, as in Lemma 11. If $b_{2}(X)=1$, then $X$ is diffeomorphic to a complex line bundle of negative degree over $\mathbb{C P}_{1}$, in such a manner that the zero section corresponds to a holomorphic curve $\mathbb{C P}_{1} \subset X$. In particular, the intersection form of $X$ is $(-k)$ for some integer $k \geq 1$, and the group $\Gamma$ at infinity is the cyclic group $\mathbb{Z}_{k}$.

Proof. By flowing along the gradient of a suitable Morse function $a_{1} t_{1}+a_{2} t_{2}$, the proof of Proposition 16 shows that $X$ is diffeomorphic to a tubular neighborhood of a single holomorphically embedded $\mathbb{C P}_{1}$ :

\footnotetext{
${ }^{3}$ This could also be proved by instead using a result of Anderson [2] Corollary 3.9].
} 


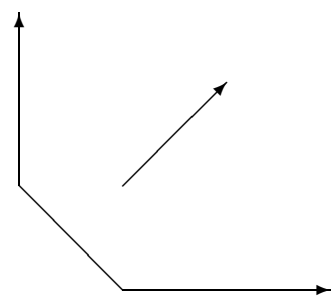

Since $X$ has negative intersection form by Proposition 13 the claim follows.

Lemma 19. Let $\left(X, g_{\infty}\right)$ be a deepest bubble, as in Lemma 11, If $b_{2}(X)=2$, then $X$ is diffeomorphic to the 4-manifold obtained by plumbing together two complex line bundles over $\mathbb{C P}_{1}$. Moreover, there is a basis for $H_{2}(X, \mathbb{Z})$, represented by a pair of totally geodesic and holomorphic $\mathbb{C P}_{1}$ 's, in which the intersection form becomes

$$
\left(\begin{array}{cc}
-k & 1 \\
1 & -\ell
\end{array}\right)
$$

for some positive integers $k \geq 2$ and $\ell \geq 1$. Finally, the group $\Gamma$ at infinity is the cyclic group $\mathbb{Z}_{k \ell-1}$.

Proof. By following the gradient lines of a suitable Morse function $a_{1} t_{1}+a_{2} t_{2}$, the proof of Proposition 16] shows that $X$ is diffeomorphic to a neighborhood of a pair of holomorphically embedded $\mathbb{C P}_{1}$ 's:

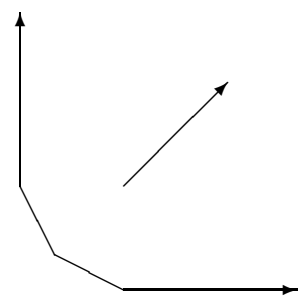

Since this neighborhood can be obtained by plumbing together the normal bundles of these $\mathbb{C P}_{1}$ 's, the intersection form certainly is given by a matrix of the above form for some $k, \ell \in \mathbb{Z}$. But $X$ has negative intersection form by Proposition 13 , so we must have $-k<0,-\ell<0$, and $-k-\ell+2<0$, as may be seen by taking the self-intersections of the two generators and their sum. Thus $k$ and $\ell$ are both positive, and one of them (without loss of generality, $k$ ) must be large than 1 .

Now the 3-manifold $S^{3} / \Gamma$ must be diffeomorphic to any level set of the Morse function occurring above the highest critical point, and our plumbing picture says that this 3-manifold can therefore be obtained from the disjoint union of $S^{3} / \mathbb{Z}_{k}$ and $S^{3} / \mathbb{Z}_{\ell}$, thought of as principal circle bundles over the two $\mathbb{C P}_{1}$ 's, by deleting a trivialized neighborhood of a fiber in each and then identifying the resulting $S^{1} \times S^{1}$ boundaries via an interchange of the factors. It follows that $S^{3} / \Gamma$ can be constructed by gluing together two solid tori $S^{1} \times D^{2}$ along their boundaries $S^{1} \times S^{1}=\mathbb{R}^{2} / \mathbb{Z}^{2}$ via

$$
\left(\begin{array}{cc}
-k & 1 \\
1 & 0
\end{array}\right)^{-1}\left(\begin{array}{ll}
0 & 1 \\
1 & 0
\end{array}\right)\left(\begin{array}{cc}
-\ell & 1 \\
1 & 0
\end{array}\right)=\left(\begin{array}{cc}
-\ell & 1 \\
1-k \ell & k
\end{array}\right) \in G L(2, \mathbb{Z}) .
$$


Thus a meridian of one torus becomes a circle of slope $\ell /(k \ell-1)$ on the other, and the 3 -manifold at infinity is therefore a Lens space $L(k \ell-1, \ell)$, with fundamental group $\Gamma \cong \mathbb{Z}_{k \ell-1}$.

\section{Obstructions to BubBling}

In light of the information gleaned from 93 , the curvature of bilaterally symmetric extremal Kähler metrics on $M=\mathbb{C P}_{2} \# 2 \overline{\mathbb{C P}}_{2}$ is rather tightly constrained, at least when $x \in\left(0, x_{0}\right]$; indeed, such metrics have

$$
\frac{1}{32 \pi^{2}} \int s^{2} d \mu=\mathcal{A}([\omega])<8
$$

if $x=\varepsilon / \beta$ is in this range. However, the Kähler condition implies that

$$
\frac{1}{8 \pi^{2}} \int_{M}\left(\frac{s^{2}}{4}-|\stackrel{\circ}{r}|^{2}\right) d \mu=c_{1}^{2}(M)=7
$$

so any of these extremal Kähler metrics actually has

$$
\int_{M}|\stackrel{r}{r}|^{2} d \mu<8 \pi^{2}
$$

By the signature formula, we also have

$$
\int_{M}\left|W_{-}\right|^{2} d \mu=-12 \pi^{2} \tau(M)+\int_{M}\left|W_{+}\right|^{2} d \mu=12 \pi^{2}+\int_{M} \frac{s^{2}}{24} d \mu
$$

for any Kähler metric. Thus extremal Kähler metrics with $\mathcal{A}([\omega])<8$ also satisfy

$$
\frac{1}{4 \pi^{2}} \int_{M}\left|W_{-}\right|^{2} d \mu<3+\frac{8 \cdot 32 \pi^{2}}{24 \cdot 4 \pi^{2}}=\frac{17}{3}
$$

implying, in particular, that

$$
\int_{M}\left|W_{-}\right|^{2} d \mu<23 \pi^{2}
$$

We will now use this and similar knowledge to prove, in stages, that curvature bubbling does not occur for sequences of such metrics.

One of the tools we will use repeatedly is a variant of the Gauss-Bonnet formula. If $\left(X, g_{\infty}\right)$ is any ALE 4-manifold with group $\Gamma$ at infinity, then the corrected form 4 of the Gauss-Bonnet formula reads

$$
\frac{1}{8 \pi^{2}} \int_{X}\left(\frac{s^{2}}{24}+\left|W_{+}\right|^{2}+\left|W_{-}\right|^{2}-\frac{|\stackrel{r}{ }|^{2}}{2}\right) d \mu_{g_{\infty}}=\chi(X)-\frac{1}{|\Gamma|}
$$

where $\chi$ is the topological Euler characteristic of the non-compact manifold and $|\Gamma|$ is the order of the group. When $\left(X, g_{\infty}\right)$ is scalar-flat Kähler, this simplifies to become

$$
\frac{1}{8 \pi^{2}} \int_{X}\left(\left|W_{-}\right|^{2}-\frac{\mid \stackrel{\circ}{2}}{2}\right) d \mu_{g_{\infty}}=\chi(X)-\frac{1}{|\Gamma|} .
$$

Our first key observation is that our deepest bubbles must necessarily have $\Gamma \neq\{1\}$.

\footnotetext{
${ }^{4}$ This may be proved by Chern's method [21]; namely, if we choose an asymptotically outward pointing vector field, the Gauss-Bonnet integral counts the number of zeroes of the vector field plus a boundary integral which would contribute -1 in Euclidean space.
} 
Lemma 20. Let $g_{i}$ be a sequence of unit volume bilaterally symmetric extremal Kähler metrics on $M=\mathbb{C P}_{2} \# 2 \overline{\mathbb{C P}}_{2}$ with $\mathcal{A}\left(g_{i}\right)<8-\delta$ for some $\delta>0$, and suppose that sectional curvatures are not uniformly bounded for this sequence. Let $\left(X, g_{\infty}\right)$ be a deepest bubble extracted by rescaling a subsequence at points of maximal curvature. Then, at infinity, the ALE scalar-flat Kähler manifold $\left(X, g_{\infty}\right)$ is asymptotic to $\mathbb{R}^{4} / \Gamma$ for some $\Gamma \neq\{1\}$. That is, $\left(X, g_{\infty}\right)$ cannot be strictly asymptotically Euclidean.

Proof. Suppose we had such a bubble with $\Gamma=1$. Then, by Propositions 12 and 13. the one-point compactification $\hat{X}=X \cup\{\infty\}$ of $X$ is a compact anti-self-dual 4 -manifold with negative intersection form. The signature formula

$$
\tau(\hat{X})=\frac{1}{12 \pi^{2}} \int_{\hat{X}}\left(\left|W_{+}\right|^{2}-\left|W_{-}\right|^{2}\right) d \mu_{\hat{g}}
$$

and the conformal invariance of $\mathcal{W}$ therefore give us

$$
\int_{X}\left|W_{-}\right|^{2} d \mu_{g_{\infty}}=12 \pi^{2} b_{2}(X)
$$

On the other hand, Lemma 14 tells us that $b_{1}=b_{3}=0$, so (9) with $|\Gamma|=1$ becomes

$$
\int_{X}\left(\left|W_{-}\right|^{2}-\frac{|r|^{2}}{2}\right) d \mu_{g_{\infty}}=8 \pi^{2} b_{2}(X)
$$

and we therefore conclude that

$$
\int_{X}|\stackrel{r}{ }|^{2} d \mu_{g_{\infty}}=8 \pi^{2} b_{2}(X)
$$

But our assumptions imply that

$$
\limsup _{i \rightarrow \infty} \int_{M} \mid r^{2} d \mu_{g_{i}} \leq 8 \pi^{2}(1-\delta)
$$

and Lemma 11 tells us that

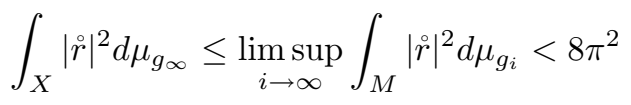

so we must have $b_{2}(X)=0$. But this now implies that both $\stackrel{\circ}{r}$ and $W_{-}$vanish identically, forcing $g_{\infty}$ to be flat. However, this is impossible, since $\left(X, g_{\infty}\right)$ is a deepest bubble. Thus deepest bubbles with $\Gamma=\{1\}$ cannot arise in the present context.

Similar reasoning gives us

Lemma 21. Let $g_{i}$ and $\left(X, g_{\infty}\right)$ be as in Lemma 20. Then $X$ is diffeomorphic to a region of $M$ which is invariant under $F_{1} \leftrightarrow F_{2}$, and this $\mathbb{Z}_{2}$-action induces a holomorphic isometric involution of $\left(X, g_{\infty}\right)$.

Proof. If $\left(X, g_{\infty}\right)$ is a deepest bubble arising as in Lemma 20, we now know that $\Gamma \neq\{1\}$, and hence $|\Gamma| \geq 2$. Moreover, $b_{1}(X)=b_{3}(X)=0$ by Lemma 14 and $b_{2} \neq 0$ by Lemma 17. Hence $\chi(X) \geq 2$, and

$$
\int_{X}\left|W_{-}\right|^{2} d \mu \geq 8 \pi^{2}\left(2-\frac{1}{2}\right) \geq 12 \pi^{2} .
$$

Since inequality (8) tells us that we have $<23 \pi^{2}$ worth of $\left\|W_{-}\right\|^{2}$ to bubble away, Lemma 15 therefore shows that $F_{1} \leftrightarrow F_{2}$ must induce an isometry of $X$ and that $X$ 
is actually diffeomorphic to a region of $M$ which is invariant under the corresponding $\mathbb{Z}_{2 \text {-action. }}$

Lemma 22. Let $g_{i}$ and $\left(X, g_{\infty}\right)$ be as in Lemma 20. If $b_{2}(X)=2$, then $\Gamma \cong \mathbb{Z}_{3}$, and $X$ has intersection form

$$
\left(\begin{array}{rr}
-2 & 1 \\
1 & -2
\end{array}\right)
$$

Proof. In conjunction with inequality (8), Lemma 11 tells us that

$$
\frac{17}{6}>\frac{1}{8 \pi^{2}} \int_{M}\left|W_{-}\right|^{2} d \mu \geq 1+b_{2}(X)-\frac{1}{|\Gamma|}
$$

so that

$$
\frac{11}{6}+\frac{1}{|\Gamma|}>b_{2}(X)
$$

When $b_{2}(X)=2$, we thus have $|\Gamma| \leq 5$.

But when $b_{2}(X)=2$, Lemma 19 tells us that the intersection form is

$$
\left(\begin{array}{cc}
-k & 1 \\
1 & -\ell
\end{array}\right)
$$

for some $k \geq 2, \ell \geq 1$, and that $\Gamma=\mathbb{Z}_{k \ell-1}$. But Lemma 21 tells us that we have a $\mathbb{Z}_{2}$-action which interchanges the two totally geodesic $\mathbb{C P}_{1}$ 's which generate $H^{2}(X, \mathbb{Z})$. The intersection form must therefore be given by

$$
\left(\begin{array}{cc}
-k & 1 \\
1 & -k
\end{array}\right)
$$

for some $k \geq 2$, and $\Gamma=\mathbb{Z}_{k^{2}-1}$. But we have also just seen that $|\Gamma| \leq 5$, so it follows that $k=2$ is the only possibility.

Lemma 23. Let $g_{i}$ and $\left(X, g_{\infty}\right)$ be as in Lemma 20. If $b_{2}(X)=1$, then $X$ must be diffeomorphic to the line bundle of degree -2 or -3 over $\mathbb{C P}_{1}$.

Proof. By Lemma 18, $X$ must be diffeomorphic to the line bundle of degree $-k$ over $\mathbb{C P}_{1}$ for some $k>0$. If $C$ denotes the homology class of the zero section, the Poincaré dual of $c_{1}$ is the rational homology class $[(k-2) / k] C$, and it follows that the limit metric satisfies

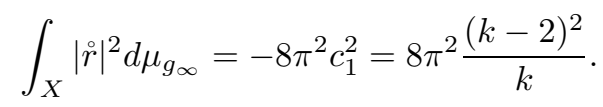

Since this number must be less that $8 \pi^{2}$ by Lemma 11, it follows that $k=2$ or 3 .

Thus, in light of Lemmas 14, 17, 22, and 23, only three cases still remain:

(i) $b_{2}(X)=1, \Gamma=\mathbb{Z}_{3}$;

(ii) $b_{2}(X)=1, \Gamma=\mathbb{Z}_{2}$; and

(iii) $b_{2}(X)=2, \Gamma=\mathbb{Z}_{3}$.

The first of these, however, is easy to eliminate:

Lemma 24. In the situation of Lemma 20, $X$ cannot be as in case (i) above. 
Proof. Suppose $X$ were as in case (i). Then, by Lemmas 21 and $23, M=\mathbb{C P}_{2} \# 2 \overline{\mathbb{C P}}_{2}$ would contain a smoothly embedded 2 -sphere $S$ of self-intersection -3 whose homology class was invariant under $F_{1} \leftrightarrow F_{2}$. This $\mathbb{Z}_{2}$-invariance of $[S] \in H_{2}(M, \mathbb{Z})$ would then allow us to express this homology class as

$$
[S]=m F_{1}+m F_{2}+n E
$$

for some integers $m$ and $n$, and the self-intersection condition would then become

$$
-3=2 m^{2}-n^{2} .
$$

But reducing this mod 3 gives us

$$
m^{2}+n^{2} \equiv 0 \bmod 3
$$

Since $m^{2}, n^{2} \equiv 0$ or $1 \bmod 3$, this can only happen if $m, n \equiv 0 \bmod 3$. But now setting $m=3 j, n=3 k$, we then have

$$
-1=6 j^{2}-3 k^{2}
$$

and reducing mod 3 again then yields a contradiction. Thus case (i) cannot arise.

Eliminating the remaining two cases is not much harder but does use considerably more of the information available to us.

Lemma 25. In the situation of Lemma 20, $X$ cannot be as in either of cases (ii) or (iii) above.

Proof. Since the limit metric $g_{\infty}$ on $X$ is by construction a pointed limit of larger and larger rescalings of the metrics $g_{i}$, the generators of $H_{2}(X, \mathbb{Z})$ must arise from smooth 2-spheres $S_{i} \subset M$ whose areas with respect to the $g_{i}$ tend to zero as $i \rightarrow \infty$. In case (ii), let $S_{i}$ be the smooth 2 -sphere corresponding to the zero section $\mathbb{C P}_{1}$; in case (iii), let $S_{i}$ be a 2 -sphere corresponding to one of the two $\mathbb{C P}_{1}$ generators and $\tilde{S}_{i}$ be its reflection under $F_{1} \leftrightarrow F_{2}$. Now any of our unit-volume bilaterally symmetric Kähler classes $\left[\omega_{i}\right]$ is of the form

$$
\left[\omega_{i}\right]=\frac{\left(1+x_{i}\right)\left(F_{1}+F_{2}\right)-x_{i} E}{\sqrt{1+2 x_{i}+\frac{x_{i}^{2}}{2}}}
$$

and has $\mathcal{A}\left([\omega]_{i}\right)=f\left(x_{i}\right)<8$. Since $\lim _{x \rightarrow \infty} f(x)=9$, we have $x_{i} \in(0, K)$ for some fixed upper bound $K$. Choose some $i$ large enough so that the associated embedded 2-sphere $S_{i} \subset M$ has area $<(K+1)^{-1}$ with respect to $g_{i}$. In case (ii), we then set $\Sigma=\left[S_{i}\right] \in H^{2}(M, \mathbb{Z})$, while in case (iii), we set $\Sigma=\left[S_{i}\right]+\left[\tilde{S}_{i}\right]$. Since $\Sigma$ is then either represented by $S_{i}$ or by $S_{i}$ plus its reflection $\tilde{S}_{i}$, we then have

$$
\left|\left[\omega_{i}\right] \cdot \Sigma\right| \leq 2 \operatorname{area}\left(S_{i}\right)<\frac{2}{K+1}
$$

by Wirtinger's inequality.

Since the homology class $\Sigma$ is $\mathbb{Z}_{2}$-invariant, we have

$$
\Sigma=j F_{1}+j F_{2}+k E
$$

for some integers $j$ and $k$. But we have arranged that $\Sigma^{2}=-2$ in either case (ii) or case (iii), so we obtain

$$
-2=2 j^{2}-k^{2}
$$


and $k \neq 0$ is therefore even, while $j$ is odd. Setting $k=2 \ell$ for some integer $\ell \neq 0$, we therefore have

$$
-1=j^{2}-2 \ell^{2}
$$

and hence

$$
\left(\frac{j}{\ell}\right)^{2}=2-\frac{1}{\ell^{2}}
$$

In particular, this tells us that

$$
\left|\frac{j}{\ell}\right| \geq 1
$$

so $j$ and $j+\ell$ cannot have opposite signs, and $j \neq 0$. Hence

$$
\begin{aligned}
\left|\left[\omega_{i}\right] \cdot \Sigma\right| & =\frac{\left|2 j\left(1+x_{i}\right)+k x_{i}\right|}{\sqrt{1+2 x_{i}+\frac{x_{i}^{2}}{2}}}=2 \frac{\left|j+(j+\ell) x_{i}\right|}{\sqrt{1+2 x_{i}+\frac{x_{i}^{2}}{2}}} \\
& >\frac{2}{\sqrt{1+2 x_{i}+\frac{x_{i}^{2}}{2}}}>\frac{2}{1+x_{i}}>\frac{2}{1+K}
\end{aligned}
$$

since $x_{i} \in(0, K)$. Hence (10) implies the glaring contradiction

$$
\frac{2}{K+1}>\left|\left[\omega_{i}\right] \cdot \Sigma\right|>\frac{2}{K+1},
$$

and it follows that cases (ii) and (iii) never actually arise.

Since all possible deepest bubbles have thus been excluded, no bubbling can occur, and Theorem 10 therefore implies the following:

Proposition 26. Let $g_{i}$ be a sequence of unit-volume bilaterally symmetric extremal Kähler metrics on $(M, J)=\mathbb{C P}_{2} \# 2 \overline{\mathbb{C P}}_{2}$ such that the corresponding Kähler classes $\left[\omega_{i}\right]$ all satisfy $\mathcal{A}\left(\left[\omega_{i}\right]\right) \leq 8-\delta$ for some $\delta>0$. Then there is a subsequence $g_{i_{j}}$ of metrics and a sequence of diffeomorphisms $\Phi_{j}: M \rightarrow M$ such that $\Phi_{j}^{*} g_{i_{j}}$ converges in the smooth topology to an extremal Kähler metric on the smooth 4-manifold $M$ compatible with some complex structure $\tilde{J}=\lim _{j \rightarrow \infty} \Phi_{j *} J$.

\section{The proof of Theorem A}

In the previous section, we saw that sequences of bilaterally symmetric extremal Kähler metrics with $\mathcal{A}<8-\delta$ necessarily have subsequences which converge as smooth metrics. We now use this to study the set of Kähler classes which admit extremal Kähler metrics.

For any positive real number $x>0$, let $[\omega]_{x}$ denote the Kähler class $(1+x)\left(F_{1}+\right.$ $\left.F_{2}\right)-x E$ on $(M, J)=\mathbb{C P}_{2} \# 2 \overline{\mathbb{C P}}_{2}$, and let us once again consider the function $f(x)=\mathcal{A}\left([\omega]_{x}\right)$ studied in $\$ 3$ Set $L$ denote the smallest positive number in $f^{-1}(8)$, so that $(0, L)$ is a connected component5 of $f^{-1}[(0,8)] \cap \mathbb{R}^{+}$.

Theorem 27. For every $x \in(0, L),[\omega]_{x}=(1+x)\left(F_{1}+F_{2}\right)-x E$ is the Kähler class of an extremal Kähler metric on the complex surface $M=\mathbb{C P}_{2} \# 2 \overline{\mathbb{C P}}_{2}$ obtained by blowing up $\mathbb{C P}_{2}$ at two distinct points.

${ }^{5}$ We are actually convinced that there are no other connected components, but there is no pressing need to try to prove this here! 
Proof. Consider the subset $\mathcal{X}$ of the interval $(0, L)$ consisting of those $x$ for which $[\omega]_{x}$ contains an extremal Kähler metric. Then $\mathcal{X}$ is non-empty [4] and open [44. Since $(0, L)$ is connected, it therefore suffices to show that $\mathcal{X}$ is also closed.

To this end, consider a sequence $x_{i} \in \mathcal{X}$ which converges to some $x \in(0, L)$. Consider the corresponding extremal Kähler metrics $g_{i}$, which have volume $[\omega]_{x_{i}}^{2} / 2=$ $1+2 x_{i}+x_{i}^{2} / 2 \rightarrow 1+2 x+x^{2} / 2=[\omega]_{x}^{2} / 2$. By rescaling these to unit volume, applying Proposition 26. and then rescaling back, there must exist a subsequence $g_{i_{j}}$ of the $g_{i}$ and a sequence of diffeomorphisms $\Phi_{j}$ such that the pull-backs $\Phi_{j}^{*} g_{i_{j}}$ smoothly converge to a metric $g$ on $M$. Now recall that each of the metrics $g_{i}$ is toric, for a fixed action of the 2-torus on $M$. Choose a fixed point $p$ of this action, choose a decomposition of the tangent space $T_{p}$ into a direct sum $L_{1} \oplus L_{2}$ into two complex lines which diagonalize the induced action, and consider its images $\Phi_{j}(p)$ under these diffeomorphisms. Since $M$ is compact, we may assume that these points converge to a point $\hat{p}$ in $M$; similarly, by again passing to a subsequence, we may also assume that the images of the orthogonal subspaces $L_{1}, L_{2} \subset T_{p} M$ converge to give an orthogonal decomposition of $T_{p} M$. Once this is done, we then obtain a limit isometric action of the 2-torus on $(M, g)$ by pushing forward the corresponding rotations of $T_{p} M$ and conjugating with the exponential map of $g$. Since the push-forwards $\Phi_{j *} J$ converge to a complex structure $\tilde{J}$ which is parallel with respect to $g$, we moreover conclude that this limit torus action is holomorphic with respect to $\tilde{J}$.

Now each of the holomorphic curves $F_{1}, F_{2}$ and $E$ in $\mathbb{C P}_{2} \# 2 \overline{\mathbb{C P}}_{2}$ is left fixed by the isometric action of some circle in the 2-torus, so each is totally geodesic with respect to the $g_{i}$. By looking at the corresponding fixed point sets of the limiting action of circle subgroups, we can therefore find totally geodesic 2 -spheres in $(M, g)$ which are the limits of the images of these submanifolds. These limit 2-spheres are moreover holomorphic curves with respect to $\tilde{J}$ and have the same homological intersection numbers as the original curves $F_{1}, F_{2}$, and $E$. By blowing down the image of $E$ and applying surface classification, we thus conclude that $(M, \tilde{J})$ is biholomorphic to the blow-up of $\mathbb{C P}_{1} \times \mathbb{C P}_{1}$ at a point. Moreover, since the areas of these totally geodesic 2-spheres are the limits of the areas of the corresponding $\mathbb{C P}_{1}$ 's with respect to the $g_{i_{j}}$, the Kähler class of $g$ on $(M, \tilde{J})$ must be the limit of the $[\omega]_{i_{j}}$. Thus there exists a diffeomorphism $\Phi: M \rightarrow M$ such that $\Phi^{*} \tilde{J}=J$ and such that $\Phi^{*} g$ becomes an extremal Kähler metric with Kähler class $[\omega]_{x}$. This shows that $x \in \mathcal{X}$. Thus $\mathcal{X}$ is closed, and the result follows.

Now Lemma 3 tells us that $x_{0} \in(0, L)$. It therefore follows that

$$
[\omega]_{x_{0}}=\left(1+x_{0}\right)\left(F_{1}+F_{2}\right)-x_{0} E
$$

is the Kähler class of an extremal Kähler metric $g$. However, Corollary 8 then tells us that the conformally related metric $h=s^{-2} g$ is Einstein and defined on all of $M$. We have therefore proved the existence of an Einstein metric on $\mathbb{C P}_{2} \# 2 \overline{\mathbb{C P}}_{2}$ which is conformally Kähler and therefore Hermitian, precisely as claimed by Theorem $\mathrm{A}$.

\section{Concluding Remarks}

While we have proved the existence of the Einstein metrics promised by Theorem A and its corollaries, we have not proved that such metrics are necessarily unique up to rescaling. On the other hand, in light of [19], this would follow [41 if the 
critical points of $\mathcal{A}$ could simply be shown to form a unique ray in the Kähler cone. Extensive computer calculations by Gideon Maschler [53] provide overwhelming evidence to this effect and could arguably be called a "computer-assisted proof" of this assertion. Nonetheless, it might be wiser to simply treat Maschler's calculations as strong circumstantial evidence, rather than as a definitive proof. In any case, a conceptual proof more accessible to the human mind would be a prerequisite to any claim that we really understand this phenomenon. One valiant attempt in this regard was made by Simanca and Stelling [64], who calculated the Hessian of the functional and concluded that all critical points must be local minima; but, contrary to what is tacitly assumed in their paper, this alone does not logically suffice to show that the critical ray is actually unique. We would therefore like to draw attention to this important gap in our knowledge, in the hope that some interested reader will be inspired to provide a definitive solution to the uniqueness problem.

Of course, the results of this paper also prove the existence of extremal Kähler metrics in a whole range of bilaterally symmetric Kähler classes on $\mathbb{C P}_{2} \# 2 \overline{\mathbb{C P}}_{2}$ other than the 'target' class $x=x_{0}$ used to construct our conformally Kähler Einstein metric. For example, it is not difficult to show that $x=1$ actually lies in the interval $(0, L)$ of Theorem 27, and this implies that the first Chern class $c_{1}(M)$ is actually the Kähler class of an extremal Kähler metric. In fact, in light of Theorem 10. it seems plausible to us that every bilaterally symmetric Kähler class might be represented by such a metric, but one would certainly need to consider many more possible bubbling modes as $x \rightarrow \infty$. Nonetheless, since the results of Arezzo, Pacard, and Singer [4 do imply the existence of such metrics for all sufficiently large $x$, such a conjecture might seem quite tempting. Of course, it would also be highly desirable to understand existence for Kähler classes which do not satisfy our convenient but somewhat arbitrary condition of bilateral symmetry. However, it is not hard to check that large regions of the Kähler cone of $\mathbb{C P}_{2} \# 2 \overline{\mathbb{C P}}_{2}$ actually lie outside the controlled cone of 95 , so our method of controlling Sobolev constants, leading to Theorem 10, actually exploited the imposition of bilateral symmetry in an essential manner.

The key rôle of toric geometry in the present paper may make it seem curious that we have not consistently operated in the toric context throughout, rather than taking limits which are only then proved to be toric at the price of considerable extra effort. Since Donaldson 24 has outlined a beautiful, systematic program for the study of toric extremal Kähler manifolds, we certainly wonder if some steps in our long argument could be simplified or eliminated altogether through the adoption of a different point of view!

We would like to once again draw the reader's attention to the central rôle played by ALE scalar-flat Kähler surfaces in our proof. Although there is a considerable literature [13, 14, 17, 28, 31, 34, 35, 37, 38, 39] concerning the construction of such metrics, it is apparent that too little is still known about their classification outside the hyper-Kähler realm so thoroughly mapped out by Kronheimer [36. In general, this problem seems daunting, but in the toric case it might be feasible to prove that the only possibilities are the metrics constructed explicitly by Calderbank and Singer [17. A related problem would be to try to classify toric anti-self-dual orbifolds by extending the beautiful paper of Fujiki [26]. 
Finally, we believe that it would be interesting to extend the techniques used in this paper to construct Bach-flat Kähler metrics which are not globally conformally Einstein. Such metrics can certainly sometimes exist when $c_{1}$ fails to be positive; for example, the study of extremal Kähler metrics on Hirzebruch surfaces [33. reveals that the differentiable manifold $S^{2} \times S^{2}$ admits Bach-flat conformal structures corresponding to many different critical values of the Weyl action $\mathcal{W}$. It would certainly be interesting to see if this same phenomenon occurs for many other complex surfaces. We hope that it may prove possible to use our present methods to construct such metrics on certain other surfaces with $c_{1}^{2}>0$. In any case, the convergence of sequences of extremal Kähler metrics on complex surfaces seems destined to develop into a rich subject of broad interest, ultimately involving issues of algebro-geometric stability [23, 24, 52, 61, 62, 68] that have played no rôle at all in the present article.

\section{ACKNOWLEDGMENTS}

The first author gratefully thanks S.K. Donaldson for many enlightening discussions of extremal Kähler metrics and the Princeton mathematics department for its hospitality during the writing of this article. The second author would like to thank Michael Taylor for some helpful comments regarding regularity issues and Michael Anderson for some useful pointers concerning Gromov-Hausdorff convergence.

\section{REFERENCES}

[1] M. T. Anderson, Ricci curvature bounds and Einstein metrics on compact manifolds, J. Amer. Math. Soc., 2 (1989), pp. 455-490. MR999661 (90g:53052)

[2] - Orbifold compactness for spaces of Riemannian metrics and applications, Math. Ann., 331 (2005), pp. 739-778. MR2148795 (2006c:53029)

[3] _ Canonical metrics on 3-manifolds and 4-manifolds, Asian J. Math., 10 (2006), pp. 127-163. MR 2213687(2007a:53074)

[4] C. Arezzo, F. Pacard, And M. Singer, Extremal metrics on blow ups. e-print math.DG/0701028.

[5] M. F. AтIYAн, Green's functions for self-dual four-manifolds, in Mathematical Analysis and Applications, Part A, vol. 7 of Adv. in Math. Suppl. Stud., Academic Press, New York, 1981, pp. 129-158. MR634238(83a:53029)

[6] _ Convexity and commuting Hamiltonians, Bull. London Math. Soc., 14 (1982), pp. 115. MR642416 (83e:53037)

[7] M. F. Atiyah, N. J. Hitchin, and I. M. Singer, Self-duality in four-dimensional Riemannian geometry, Proc. Roy. Soc. London Ser. A, 362 (1978), pp. 425-461. MR506229 (80d:53023)

[8] T. Aubin, Equations du type Monge-Ampère sur les variétés kählériennes compactes, C. R. Acad. Sci. Paris, 283A (1976), pp. 119-121. MR0433520 (55:6496)

[9] _ Some Nonlinear Problems in Riemannian Geometry, Springer Monographs in Mathematics, Springer-Verlag, Berlin, 1998. MR 1636569 (99i:58001)

[10] R. BACH, Zur Weylschen Relativitätstheorie und der Weylschen Erweiterung des Krümmungstensorbegriffs, Math. Zeitschr., 9 (1921), pp. 110-135. MR1544454

[11] W. Barth, C. Peters, and A. V. De Ven, Compact Complex Surfaces, Springer-Verlag, 1984. MR749574 (86c:32026)

[12] A. Besse, Einstein Manifolds, Springer-Verlag, 1987. MR867684 (88f:53087)

[13] E. Calabi, Métriques kählériennes et fibrés holomorphes, Ann. Sci. École Norm. Sup. (4), 12 (1979), pp. 269-294. MR543218 (83m:32033)

[14] E. CAlabi, Isometric families of Kähler structures, in The Chern Symposium 1979 (Proc. Internat. Sympos., Berkeley, Calif., 1979), Springer, New York, 1980, pp. 23-39. MR609556 (83g:53044) 
[15] _ Extremal Kähler metrics, in Seminar on Differential Geometry, vol. 102 of Ann. of Math. Stud., Princeton Univ. Press, Princeton, N.J., 1982, pp. 259-290. MR645743 (83i:53088)

[16] _ Extremal Kähler metrics. II, in Differential geometry and complex analysis, Springer, Berlin, 1985, pp. 95-114. MR780039 (86h:53067)

[17] D. M. J. Calderbank and M. A. Singer, Einstein metrics and complex singularities, Invent. Math., 156 (2004), pp. 405-443. MR2052611 (2005h:53064)

[18] X. X. Chen, Space of Kähler metrics III-on the lower bound of the Calabi energy and geodesic distance, e-print math.DG/0606228.

[19] X. X. Chen and G. Tian, Uniqueness of extremal Kähler metrics, C. R. Math. Acad. Sci. Paris, 340 (2005), pp. 287-290. MR2121892 (2006h:32020)

[20] X. X. Chen and B. Weber, Moduli spaces of critical Riemannian metrics with $L^{n / 2}$ norm curvature bounds, e-print arXiv:0705.4440, 2007.

[21] S.-S. Chern, A simple intrinsic proof of the Gauss-Bonnet formula for closed Riemannian manifolds, Ann. of Math. (2), 45 (1944), pp. 747-752. MR0011027(6:106a)

[22] A. Derdziński, Self-dual Kähler manifolds and Einstein manifolds of dimension four, Compositio Math., 49 (1983), pp. 405-433. MR707181 (84h:53060)

[23] S. K. Donaldson, Scalar curvature and projective embeddings. I, J. Differential Geom., 59 (2001), pp. 479-522. MR.1916953 (2003j:32030)

[24] - Scalar curvature and stability of toric varieties, J. Differential Geom., 62 (2002), pp. 289-349. MR.1988506 (2005c:32028)

[25] R. Friedman and J. Morgan, Algebraic surfaces and Seiberg-Witten invariants, J. Alg. Geom., 6 (1997), pp. 445-479. MR.1487223 (99b:32045)

[26] A. FujIKI, Compact self-dual manifolds with torus actions, J. Differential Geom., 55 (2000), pp. 229-324. MR.1847312 (2002k:57085)

[27] A. Futaki And T. MabuChi, Uniqueness and periodicity of extremal Kähler vector fields, in Proceedings of GARC Workshop on Geometry and Topology '93 (Seoul, 1993), vol. 18 of Lecture Notes Ser., Seoul, 1993, Seoul Nat. Univ., pp. 217-239. MR.1270938 (95c:32028)

[28] G. W. Gibbons And S. W. HaWking, Classification of gravitational instanton symmetries, Comm. Math. Phys., 66 (1979), pp. 291-310. MR535152 (80d:83025)

[29] C. D. Hill and M. TAYlor, Integrability of rough almost complex structures, J. Geom. Anal., 13 (2003), pp. 163-172. MR.1967042 (2003m:32023)

[30] N. J. Hitchin, On compact four-dimensional Einstein manifolds, J. Differential Geom., 9 (1974), pp. 435-442. MR0350657 (50:3149)

[31] , Polygons and gravitons, Math. Proc. Cambridge Philos. Soc., 85 (1979), pp. 465-476. MR520463 (80h:53047)

[32] _ Kählerian twistor spaces, Proc. London Math. Soc. (3), 43 (1981), pp. 133-150. MR623721 (84b:32014)

[33] A. D. Hwang And S. R. Simanca, Extremal Kähler metrics on Hirzebruch surfaces which are locally conformally equivalent to Einstein metrics, Math. Ann., 309 (1997), pp. 97-106. MR.1467648 (98f:58056)

[34] D. D. JoYCE, Explicit construction of self-dual 4-manifolds, Duke Math. J., 77 (1995), pp. 519-552. MR.1324633 (96d:53049)

[35] P. B. Kronheimer, Instantons gravitationnels et singularités de Klein, C. R. Acad. Sci. Paris Sér. I Math., 303 (1986), pp. 53-55. MR851268(88a:53043)

[36] _ A Torelli-type theorem for gravitational instantons, J. Differential Geom., 29 (1989), pp. 685-697. MR992335 (90d:53056)

[37] C. LeBrun, Counter-examples to the generalized positive action conjecture, Comm. Math. Phys., 118 (1988), pp. 591-596. MR962489 (89f:53107)

[38] — Explicit self-dual metrics on $\mathbb{C P}_{2} \# \cdots \# \mathbb{C P}_{2}$, J. Differential Geom., 34 (1991), pp. 223-253. MR.1114461 (92g:53040)

[39] _ Twistors, Kähler manifolds, and bimeromorphic geometry. I, J. Amer. Math. Soc., 5 (1992), pp. 289-316. MR1137098 (92m:32052)

[40] _ Anti-self-dual metrics and Kähler geometry, in Proceedings of the International Congress of Mathematicians, Vol. 1, 2 (Zürich, 1994), Basel, 1995, Birkhäuser, pp. 498-507. MR:1403950 (97h:53049)

[41] _ On the scalar curvature of complex surfaces, Geom. Funct. Anal., 5 (1995), pp. 619628. MR.1339820 (97a:53112) 
[42] _ Einstein metrics on complex surfaces, in Geometry and Physics (Aarhus, 1995), Dekker, New York, 1997, pp. 167-176. MR.1423163 (97j:53048)

[43] _ Twistors for tourists: a pocket guide for algebraic geometers, in Algebraic geometry-Santa Cruz 1995, vol. 62 of Proc. Sympos. Pure Math., Amer. Math. Soc., Providence, RI, 1997, pp. 361-385. MR 1492540 (99i:32038)

[44] C. LeBrun and S. R. Simanca, On the Kähler classes of extremal metrics, in Geometry and Global Analysis (Sendai, 1993), Tohoku Univ., Sendai, 1993, pp. 255-271. MR1361191 (96h:58037)

[45] C. LeBrun and S. R. Simanca, Extremal Kähler metrics and complex deformation theory, Geom. Funct. Anal., 4 (1994), pp. 298-336. MR1274118 (95k:58041)

[46] J. Lee and T. Parker, The Yamabe problem, Bull. Am. Math. Soc., 17 (1987), pp. 37-91. MR888880 (88f:53001)

[47] J. LELONG-FERRAND, Transformations conformes et quasiconformes des variétés riemanniennes; application à la démonstration d'une conjecture de A. Lichnerowicz, C. R. Acad. Sci. Paris Sér. A-B, 269 (1969), pp. A583-A586. MR0254782 (40:7989)

[48] P. Li And L.-F. TAM, Harmonic functions and the structure of complete manifolds, J. Differential Geom., 35 (1992), pp. 359-383. MR.1158340 (93b:53033)

[49] — Green's functions, harmonic functions, and volume comparison, J. Differential Geom., 41 (1995), pp. 277-318. MR.1331970 (96f:53054)

[50] J.-L. LiONS AND E. MAGENes, Non-homogeneous boundary value problems and applications. Vol. I, Springer-Verlag, New York, 1972. Translated from the French by P. Kenneth, Die Grundlehren der mathematischen Wissenschaften, Band 181. MR0350177 (50:2670)

[51] A.-K. Liu, Some new applications of general wall crossing formula, Gompf's conjecture and its applications, Math. Res. Lett., 3 (1996), pp. 569-585. MR.1418572 (97k:57038)

[52] T. Mabuchi, Stability of extremal Kähler manifolds, Osaka J. Math., 41 (2004), pp. 563-582. MR.2107663 (2005m:32046)

[53] G. Maschler, Distinguished Kähler Metrics and Equivariant Cohomological Invariants, $\mathrm{PhD}$ thesis, State University of New York at Stony Brook, 1997. http://www.mathcs. emory.edu/ gm/bss4forC.pdf.

[54] Y. Matsushima, Sur la structure du groupe d'homéomorphismes d'une certaine varieté Kählérienne, Nagoya Math. J., 11 (1957), pp. 145-150. MR0094478 (20:995)

[55] M. ОватA, The conjectures on conformal transformations of Riemannian manifolds, J. Differential Geometry, 6 (1971/72), pp. 247-258. MR0303464 (46:2601)

[56] H. Ohta And K. Ono, Notes on symplectic 4-manifolds with $b_{2}^{+}=1$. II, Internat. J. Math., 7 (1996), pp. 755-770. MR1417784 (97k:57042)

[57] D. PAGE, A compact rotating gravitational instanton, Phys. Lett., 79B (1979), pp. 235-238.

[58] R. Penrose, Nonlinear gravitons and curved twistor theory, General Relativity and Gravitation, 7 (1976), pp. 31-52. MR0439004 (55:11905)

[59] R. Penrose and W. Rindler, Spinors and space-time. Vol. 2, Cambridge University Press, Cambridge, 1986. Spinor and twistor methods in space-time geometry. MR838301 (88b:83003)

[60] M. Ponteconvo, Uniformization of conformally flat Hermitian surfaces, Differential Geom. Appl., 2 (1992), pp. 295-305. MR.1245329 (94k:32052)

[61] J. Ross, Unstable products of smooth curves, Invent. Math., 165 (2006), pp. 153-162. MR2221139(2007c:32027)

[62] J. Ross And R. Thomas, An obstruction to the existence of constant scalar curvature Kähler metrics, J. Differential Geom., 72 (2006), pp. 429-466. MR2219940(2007c:32028)

[63] S. R. Simanca, Strongly extremal Kähler metrics, Ann. Global Anal. Geom., 18 (2000), pp. 29-46. MR1739523 (2001b:58023)

[64] S. R. Simanca And L. D. Stelling, Canonical Kähler classes, Asian J. Math., 5 (2001), pp. 585-598. MR1913812 (2003f:32031)

[65] J. A. Thorpe, Some remarks on the Gauss-Bonnet formula, J. Math. Mech., 18 (1969), pp. 779-786. MR0256307 (41:963)

[66] G. Tian, On Calabi's conjecture for complex surfaces with positive first Chern class, Inv. Math., 101 (1990), pp. 101-172. MR.1055713 (91d:32042)

[67] — Moduli space of extremal Kähler metrics, unpublished notes, SUNY Stony Brook, 1992. 
[68] _ Kähler-Einstein metrics with positive scalar curvature, Invent. Math., 130 (1997), pp. 1-37. MR.1471884 (99e:53065)

[69] G. Tian and J. Viaclovsky, Volume growth, curvature decay, and critical metrics, e-print math.DG/0612491.

[70] _ Bach-flat asymptotically locally Euclidean metrics, Invent. Math., 160 (2005), pp. 357-415. MR:2138071 (2006b:53050)

[71] _ Moduli spaces of critical Riemannian metrics in dimension four, Adv. Math., 196 (2005), pp. 346-372. MR2166311(2006i:53051)

[72] G. Tian And S. T. YAU, Kähler-Einstein metrics on complex surfaces with $\mathbf{c}_{1}>0$, Comm. Math. Phys., 112 (1987), pp. 175-203. MR 904143 (88k:32070)

[73] E. Witten, Monopoles and four-manifolds, Math. Res. Lett., 1 (1994), pp. 809-822. MR.1306021 (96d:57035)

[74] S. T. YAU, Calabi's conjecture and some new results in algebraic geometry, Proc. Nat. Acad. USA, 74 (1977), pp. 1789-1799. MR0451180 (56:9467)

Department of Mathematics, University of Wisconsin-Madison, 480 Lincoln Dr, MadiSON, WISCONSIN 53706-1388

E-mail address: xiu@math.wisc.edu

Department of Mathematics, State University of New York, Stony Brook, New York 11794-3651

E-mail address: claude@math.sunysb.edu

Department of Mathematics, State University of New York, Stony Brook, New York 11794-3651

E-mail address: brweber@math.sunysb.edu 